\title{
Interaction region design driven by energy deposition
}

\author{
Roman Martin, Maria Ilaria Besana, Francesco Cerutti, Andy Langner, and Rogelio Tomás \\ CERN, CH 1211 Geneva 23, Switzerland \\ Emilia Cruz-Alaniz \\ John Adams Institute, University of Oxford, Oxford OX1 3RH, United Kingdom
}

Barbara Dalena

CEA, IRFU, SACM, Centre de Saclay, F-91191 Gif-sur-Yvette, France

(Received 24 February 2017; published 30 August 2017)

\begin{abstract}
The European Strategy Group for High Energy Physics recommends to study collider designs for the post-LHC era. Among the suggested projects there is the circular $100 \mathrm{TeV}$ proton-proton collider FCC-hh. Starting from LHC and its proposed upgrade HL-LHC, this paper outlines the development of the interaction region design for FCC-hh. We identify energy deposition from debris of the collision events as a driving factor for the layout and draft the guiding principles to unify protection of the superconducting final focus magnets from radiation with a high luminosity performance. Furthermore, we offer a novel strategy to mitigate the lifetime limitation of the first final focus magnet due to radiation load, the Q1 split.
\end{abstract}

DOI: 10.1103/PhysRevAccelBeams.20.081005

\section{INTRODUCTION}

The Future Circular Collider (FCC) study is exploring possible designs of circular colliders for the post-LHC era. Among the studied options are an energy upgrade of the LHC (HE-LHC), an $e^{+} e^{-}$collider (FCC-ee) and a hadron collider with a focus on proton-proton collisions at a centerof-mass energy of $100 \mathrm{TeV}$ and the option of heavy ion operation (FCC-hh). Like the LHC, FCC-hh is designed to accommodate two high luminosity, general purpose experiments, as well as two special-purpose experiments. The high luminosity interaction regions (IRs) will require a strongly focused beam with a small $\beta$ function $\beta^{*}$ at the interaction point (IP). At the same time they need to provide enough space for the detectors resulting in a long distance $L^{*}$ between the IP and the first quadrupole. Consequently, the high luminosity IRs will feature the largest $\beta$ functions in the whole machine, along with all the associated challenges like mechanical aperture constraints, chromaticity and dynamic aperture. Thus they will be the focus of this paper.

In the scope of this work, the same quadrupole magnet technology as in the HL-LHC is assumed, while the beam energy increases by a factor of about 7.6. Therefore the limitations on performance arising from this scaling need to be evaluated. The center-of-mass energy of $100 \mathrm{TeV}$ is

\footnotetext{
*Also at Physics Department, Humboldt University, Berlin.

Published by the American Physical Society under the terms of the Creative Commons Attribution 4.0 International license. Further distribution of this work must maintain attribution to the author(s) and the published article's title, journal citation, and DOI.
}

unprecedented and new challenges coming with it must be identified and addressed. One of the expected challenges is the radiation load in the final focus system coming from collision debris from the interaction point. The high luminosity upgrade of the LHC already requires dedicated shielding inside the final focus magnets to cope with the increased radiation load. For FCC-hh this represents a challenge as the radiation load increases with the following: (i) center-of-mass energy of the colliding beams $E_{\mathrm{cm}}$; (ii) inelastic proton-proton cross section $\sigma_{\text {inel,pp }}$; (iii) peak luminosity; and (iv) integrated luminosity.

The corresponding values are listed in Table I for LHC, HL-LHC and FCC-hh. The instantaneous luminosity drives the total power on the magnets and the peak power density in the superconducting coils. The total power is a key element for the design of the cryogenic system, needed to evacuate the heat load. An excessive peak power density in the coils can cause a magnet quench, therefore it must be well below the quench limit to guarantee stable operation of the FCC-hh accelerator. The total power towards each side of the interaction point is expected to be $43 \mathrm{~kW}$ with baseline parameters (see Table I), compared to $1 \mathrm{~kW}$ for LHC and $4.76 \mathrm{~kW}$ for HL-LHC. For ultimate parameters, the power will increase to $260 \mathrm{~kW}$. While the resulting power densities in the superconducting coils of the final focus system will depend on the geometry, magnet strengths and crossing angles, we can expect them to scale in the same order of magnitude as the total power if no additional mitigation measures are taken. The integrated luminosity will determine long term radiation damage, mainly due to the degradation of insulator material and epoxy resin used to impregnate coils, as well as the activation of the magnets. Again a rough estimate from 
TABLE I. Parameters contributing to the radiation load.

\begin{tabular}{|c|c|c|c|c|c|}
\hline & \multirow[b]{2}{*}{ LHC } & \multicolumn{2}{|c|}{ HL-LHC } & \multicolumn{2}{|c|}{ FCC-hh } \\
\hline & & Baseline & Ultimate & Baseline & Ultimate \\
\hline$E_{\mathrm{cm}}[\mathrm{TeV}]$ & 14 & \multicolumn{2}{|c|}{14} & \multicolumn{2}{|c|}{100} \\
\hline$\sigma_{\text {inel,pp }}[\mathrm{mb}]$ & $81 \pm 3^{\mathrm{a}}$ & \multicolumn{2}{|c|}{$81 \pm 3^{\mathrm{a}}$} & \multicolumn{2}{|c|}{$108^{\mathrm{b}}$} \\
\hline Peak luminosity $\left[10^{34} \mathrm{~cm}^{-2} \mathrm{~s}^{-1}\right]$ & 1 & 5 & 7.5 & 5 & 30 \\
\hline Integrated luminosity $\left[\mathrm{fb}^{-1}\right]$ & 300 & 3000 & 4000 & \multicolumn{2}{|c|}{$17500^{\mathrm{c}}$} \\
\hline
\end{tabular}

${ }^{\mathrm{a}}$ Based on TOTEM measurements at $E_{\mathrm{cm}}=7 \mathrm{TeV}$ and extrapolation using various models [1].

${ }^{\mathrm{b}}$ Value taken from [2].

${ }^{\mathrm{c}}$ Assuming 10 years of operation with baseline parameters followed by 15 years with ultimate parameters [3].

the integrated luminosity goal suggests an increase of the peak doses by 2 to 3 orders of magnitude.

\section{SCALING FROM LHC AND HL-LHC}

\section{A. LHC interaction region}

With the LHC exceeding its design peak luminosity [4] at $13 \mathrm{TeV}$ center-of-mass energy and the HL-LHC in a well advanced design phase [5], it is a good strategy for the project to take advantage of the work and experience that have been put into these projects by using their lattices and adopting them to the challenges of FCC-hh. Both LHC and HL-LHC interaction region lattices for the high luminosity experiments ATLAS and CMS are similar in their underlying layout. The LHC interaction region layout is shown in Fig. 1. Starting from the interaction point, a drift space of $L^{*}=23 \mathrm{~m}$ is reserved for the experiments. The only devices placed in this region are a beam position monitor and an absorber - target absorber secondaries (TAS) — that protects the final focus system from debris of the particle collisions. In the case of ATLAS, the TAS is located between the big wheels of the muon spectrometer (Fig. 3.1 of [6]). For CMS, the TAS is outside the detector. At $s=23 \mathrm{~m}$ the first quadrupole Q1 of the final focus triplet starts. The triplet has an overall length of $31 \mathrm{~m}$ in the LHC case and $42 \mathrm{~m}$ in HL-LHC, including drift spaces between the quadrupoles. A corrector package consisting of skew quadrupoles and higher order multipoles is installed. The triplet consists of single aperture magnets that host both beams. The triplets on both sides of the IP are powered antisymmetrically. This has the advantage that the triplet region is optically identical for both beams. Crossing angles are created by orbit correctors located in the interaction region. The crossing angle is in the vertical plane for ATLAS and in the horizontal plane for CMS. Behind the triplet, a shared aperture dipole D1 separates the two beams. After a drift of about $57 \mathrm{~m}$, the double bore dipole D2 bends the separated beams onto parallel orbits again. The four quadrupoles Q4-Q7 form the matching section that occupies the rest of the straight section. Due to the strong focusing required at Q7, it consists of two quadrupoles which are powered in series. The straight section has a total length of $537.8 \mathrm{~m}$. The dispersion suppressor (DS) design was constrained by the LEP tunnel geometry [7,8]. It consists of two cells with the dipoles having the sole purpose of guiding the orbit on the reference trajectory of the LEP tunnel while the individually powered DS quadrupoles Q8-Q10 are used to match the dispersion between arc and straight section. The end of the straight section has a nonzero dispersion due to the separation and recombination dipoles D1 and D2. In order to provide enough degrees of freedom to match all required beam parameters from the IP to the arcs, not only the matching section quadrupoles, but also the DS quadrupoles Q8-Q10 and the first three trim quadrupoles of the first arc cell QT11-QT13 are used for the matching procedure. This is necessary to match the Twiss parameters $\beta_{x}, \alpha_{x}, \beta_{y}, \alpha_{y}$, the phase advances $\mu_{x}, \mu_{y}$ and the horizontal dispersion function and its derivative $D_{x}, D_{x}^{\prime}$. In the arcs, all magnets of one family (e.g. main dipoles, focusing quadrupoles, defocusing quadrupoles) for both beams are powered in series by a single power converter per arc.

\section{B. Scaling laws}

Due to the increased energy, it is not possible to just copy the LHC or HL-LHC IR: the increased beam rigidity would

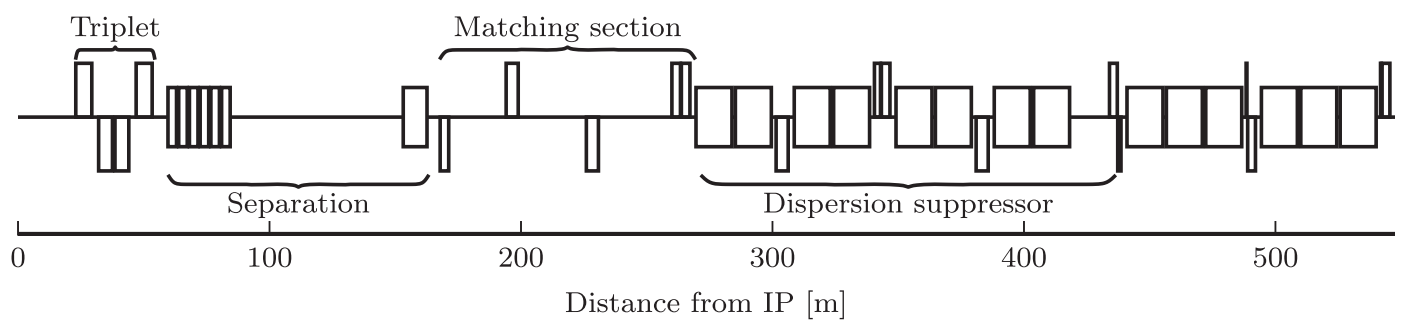

FIG. 1. Layout of the LHC interaction region. 
require a proportional increase in the magnetic field. Unfortunately, there is no prospect for such a development in magnet technology. Instead, a reasonable approach is to scale the IR length in order to cope with the decreased focusing (i.e. increased focal length) of the quadrupole magnets. In this section, the scaling of the normalized quadrupole gradient $k$ and the $\beta$ functions with respect to the length scaling factor $\alpha_{\mathrm{s}}$ are derived.

\section{Normalized gradient}

In the thin lens approximation, the normalized quadrupole strength $k$ is equal to

$$
k=\frac{1}{L_{\mathrm{Q}} f},
$$

with $L_{\mathrm{Q}}$ the quadrupole length and $f$ the focal length, both of which are proportional to $\alpha_{\mathrm{s}}$. The scaled quadrupole length $L_{\mathrm{Q}}^{\prime}=\alpha_{\mathrm{s}} \cdot L_{\mathrm{Q}}$ increases the integrated quadrupole strength while an increased focal length $f^{\prime}=\alpha_{\mathrm{s}} \cdot f$ reduces the required integrated quadrupole strength, so the normalized gradient scales like

$$
k^{\prime}=\frac{1}{L_{\mathrm{Q}}^{\prime} f^{\prime}}=\frac{1}{\alpha_{\mathrm{s}}^{2} L_{\mathrm{Q}} f}=\frac{k}{\alpha_{\mathrm{s}}^{2}},
$$

where the prime denotes the scaled parameters.

\section{Longitudinal scaling of the $\beta$ function}

The equation of motion of a particle

$$
\frac{\mathrm{d}^{2}}{\mathrm{~d} s^{2}} x(s)-k x(s)=0
$$

with $x(s)$ the transverse offset from the design orbit at the longitudinal position $s$, has the general solution

$$
x=u \sqrt{\epsilon} \cos [\Psi(s)+\psi]
$$

with $u=\sqrt{\beta(s)}$ and $\epsilon$ the emittance. This formula describes an oscillation with the phase $\Psi(s)+\psi$. Inserting this in Eq. (3) leads to the differential equation for $u$ :

$$
\frac{\mathrm{d}^{2} u}{\mathrm{~d} s^{2}}-\frac{1}{u^{3}}-k u=0
$$

Under length scaling, $\mathrm{d} s^{\prime}$ is proportional to $\alpha_{\mathrm{s}}$. Inserting this and Eq. (2) into Eq. (5) yields

$$
\frac{\mathrm{d}^{2} u^{\prime}}{\mathrm{d} s^{\prime 2}}-\frac{1}{u^{\prime 3}}-k^{\prime} u^{\prime}=\frac{1}{\alpha_{\mathrm{s}}^{2}} \frac{\mathrm{d}^{2} u^{\prime}}{\mathrm{d} s^{2}}-\frac{1}{u^{\prime 3}}-\frac{1}{\alpha_{\mathrm{s}}^{2}} k u^{\prime}=0 .
$$

Inserting the ansatz

$$
u^{\prime}=\sqrt{\alpha_{\mathrm{s}}} u,
$$

into Eq. (6) reproduces Eq. (5), i.e. the scaled beam optics are similar to the original optics. We can therefore conclude that

$$
\beta^{\prime}=\alpha_{\mathrm{s}} \beta
$$

\section{Scaling strategies}

With the general scaling laws established, we have to determine a length scaling factor $\alpha_{\mathrm{s}}$, which will provide viable optics at an increased beam energy of $50 \mathrm{TeV}$. There are different strategies, depending on the goal of the scaling.

\section{Constant beam stay clear}

The shortest possible interaction region length can be achieved by scaling for a constant beam stay clear [9]. The normalized quadrupole coefficient is given by

$$
k=\frac{e}{p} \frac{\partial B_{y}}{\partial x},
$$

with $e$ the elementary charge, $p$ the particle momentum and $\partial B_{y} / \partial x$ the quadrupole gradient. In a simplified quadrupole model, the gradient $\partial B_{y} / \partial x$ can be described as

$$
\frac{\partial B_{y}}{\partial x}=\frac{B_{\max }}{r}
$$

with $r$ the coil aperture radius and $B_{\max }$ the magnetic field at the coil aperture, which is determined by the used magnet technology. The coil aperture radius can be described as $r=N \cdot \sigma_{x}$ with $\sigma_{x}=\sqrt{\beta \epsilon_{\mathrm{n}} / \gamma}$ being the beam size and $N$ a number representing both the normalized beam separation as well as the beam stay clear required to obey the collimation hierarchy [5]. $\epsilon_{\mathrm{n}}$ represents the normalized beam emittance and $\gamma$ is the Lorentz factor. As a first guess, $N$ can be assumed constant to preserve aperture needs relative to the beam size. Thus, we can write

$$
\begin{aligned}
k & =\frac{e}{p} \frac{B_{\max }}{N \sqrt{\beta \epsilon_{\mathrm{n}} / \gamma}}, \\
k^{\prime} & =\frac{e}{p^{\prime}} \frac{B_{\max }}{N \sqrt{\beta^{\prime} \epsilon_{\mathrm{n}} / \gamma^{\prime}}},
\end{aligned}
$$

where the prime denotes the scaled lattice. According to Eq. (2) the ratio of the normalized gradients can be identified as the square of the length scaling factor $\alpha_{\mathrm{S}}$, resulting in 


$$
\alpha_{\mathrm{s}}^{2}=\frac{k}{k^{\prime}}=\frac{p^{\prime}}{p} \frac{\sqrt{\beta^{\prime}}}{\sqrt{\beta}} \frac{\sqrt{\gamma}}{\sqrt{\gamma^{\prime}}} .
$$

Both, the particle momenta and the Lorentz factors $\gamma$ scale with $\frac{50 \mathrm{TeV}}{7 \mathrm{TeV}}$ while the $\beta$ functions scale with $\alpha_{\mathrm{s}}$. With this, rearranging Eq. (13) results in a scaling factor of

$$
\alpha_{\mathrm{s}}=\left(\frac{50 \mathrm{TeV}}{7 \mathrm{TeV}}\right)^{1 / 3} \approx 2
$$

This scaling accounts neither for different normalized emittances nor for the increase of normalized separation needed due the higher number of long range beam-beam interactions.

\section{Constant gradients and apertures}

A constant beam stay clear is not the only valid option. Due to technical constraints and impedance issues, the collimation system is scaled in order to achieve collimator gaps similar to the LHC [10,11]. As the beam stay clear requirement for the interaction region is determined by the collimation system via the collimation hierarchy, it makes sense to use a scaling factor for the IR lattice that also preserves magnet apertures and gradients. Instead of using the emittance reduction at higher energies to allow for small magnet apertures, it is possible to keep the gradient $\partial B_{y} / \partial x$ in Eq. (9) - and thus the coil aperture according to Eq. (10)—constant and only apply length scaling according to the reduced focal strengths. From Eqs. (2) and (9), we can deduce

$$
\frac{k}{k^{\prime}}=\frac{p^{\prime}}{p}=\alpha_{\mathrm{s}}^{2},
$$

so the scaling factor is $\alpha_{\mathrm{s}}=\sqrt{\frac{50 \mathrm{TeV}}{7 \mathrm{TeV}}} \approx 2.67$. A major advantage of this scaling is the constant gradient, allowing to reuse existing magnet technology. As the $\beta$ functions in the triplet also scale with $\alpha_{\mathrm{s}}$ while the emittance decreases with $1 / \gamma \propto \frac{7 \mathrm{TeV}}{50 \mathrm{TeV}}=\alpha_{\mathrm{s}}^{-2}$, the beam stay clear of the scaled lattice should increase with $\sqrt{1 / \alpha_{\mathrm{s}}} \approx 1.64$.

\section{Minimum $\beta^{*}$ under length scaling}

An alternative to scaling with the beam energy is to start from a lattice and scale its length at constant energy in order to reduce the minimum $\beta$ function at the IP, $\beta^{*}{ }_{\min }$, and to increase the luminosity. This approach is complementary to a study using constant gradient point to parallel focusing [12].

In the scope of this work, $\beta_{\min }^{*}$ was assumed to be limited by the apertures of the triplet magnets. These apertures are affected by the length scaling factor $\alpha_{\mathrm{s}}$. To investigate the scaling of the $\beta^{*}$ reach with $\alpha_{\mathrm{s}}$, we first have to determine the relation of $\beta^{*}$ and the $\beta$ function in the triplet. With known $\beta, \beta^{*}$ and phase advance $\Psi$, the transfer matrix is given by

$$
M=\left(\begin{array}{cc}
\sqrt{\frac{\beta}{\beta^{*}}}\left(\cos \Psi+\alpha^{*} \sin \Psi\right) & \sqrt{\beta \beta^{*}} \sin \Psi \\
\frac{\left(\alpha^{*}-\alpha\right) \cos \Psi-\left(1+\alpha^{*} \alpha\right) \sin \Psi}{\sqrt{\beta \beta^{*}}} & \sqrt{\frac{\beta^{*}}{\beta}}(\cos \Psi-\alpha \sin \Psi)
\end{array}\right) .
$$

Specifically the matrix elements $m_{11}$ and $m_{12}$ that define the beam size are of interest here. The phase advance between the IP and any point in the triplet is $\Psi \approx \frac{\pi}{2}$ [12]. With this and $\alpha^{*}=0$, the matrix element $m_{11}$ vanishes while $m_{12} \approx \sqrt{\beta \beta^{*}}$. Thus, we can deduce

$$
\beta_{\text {triplet }} \approx m_{12}^{2} \frac{1}{\beta^{*}} .
$$

Under normal scaling, both $\beta$ and $\beta^{*}$ are proportional to the length scaling factor $\alpha_{\mathrm{s}}$ (see Sec. II B 2). Thus, from Eq. (17) we can deduce that $m_{12} \propto \alpha_{\mathrm{s}}$, which must hold true independent of $\beta^{*}$. Consequently, if $\beta^{*}$ is kept constant, the beam size at any given point in the triplet scales with $\sigma_{x} \propto \alpha_{\mathrm{s}}$.

Next, the scaling of the quadrupole aperture is examined. With the normalized gradient definition in Eq. (9) and the aperture-gradient model given by Eq. (10), we can describe the coil aperture radius with

$$
r=\frac{e}{p} \frac{B_{\max }}{k}
$$

which scales with $\alpha_{\mathrm{s}}^{2}$, since $k$ scales with $1 / \alpha_{\mathrm{s}}^{2}$ according to Eq. (2). Layers that reduce the free aperture, like the liquid helium gap (LHe), Kapton insulator, cold bore, beam screen, beam screen insulator and shielding are assumed to be constant. Together with the closed orbit uncertainty, they are included in the constant aperture reduction $r_{\text {etc }}$. It should be noted that in reality, the cold bore thickness scales with the coil aperture. This is a minor effect and can be easily incorporated in the following calculations.

Lastly, we need to scale the aperture requirement. We assume a constant beam stay clear requirement $N_{\text {bsc }}$. The crossing angle requirement due to beam-beam effects $N_{\mathrm{bb}}$ in units of beam sizes $\sigma_{x}$ depends on the number of long range beam-beam interactions $N_{\text {lr }}$ which is proportional to the distance between IP and separation dipole D1, consequently $N_{\text {lr }} \propto \alpha_{\mathrm{s}}$. According to [13], the long range beam-beam tune shift scales approximately with

$$
\Delta Q_{\mathrm{lr}} \propto \frac{N_{\mathrm{lr}}}{N_{\mathrm{bb}}^{2}} .
$$

With $N_{\text {lr }} \propto \alpha_{\mathrm{S}}$ we get a constant tune shift $\Delta Q_{\text {lr }}$ for $N_{\mathrm{bb}} \propto \sqrt{\alpha_{\mathrm{s}}}$. 
TABLE II. Parameters of the lattice options.

\begin{tabular}{|c|c|c|c|c|c|c|}
\hline & LHC & HL-LHC & \multicolumn{4}{|c|}{ FCC-hh } \\
\hline Normalized transverse emittance $\epsilon_{\mathrm{n}}[\mu \mathrm{m}]$ & 3.75 & 2.5 & \multicolumn{4}{|c|}{2.2} \\
\hline$L^{*}[\mathrm{~m}]$ & 23 & 23 & 46 & 36 & 61.5 & 45 \\
\hline Quadrupole length $L_{\mathrm{Q} 1 / \mathrm{Q} 3}[\mathrm{~m}]$ & 6.37 & 7.685 & 12.74 & 20 & 20.54 & 31.81 \\
\hline Quadrupole length $L_{\mathrm{Q} 2 \mathrm{a} / \mathrm{b}}[\mathrm{m}]$ & 5.50 & 6.577 & 11.0 & 17.5 & 17.58 & 26.37 \\
\hline Quadrupole coil aperture diameter [mm] & 70 & $150^{\mathrm{a}}$ & 62 & $100 / 115^{\mathrm{b}}$ & 140 & $205 / 248$ \\
\hline Quadrupole gradient $[\mathrm{T} / \mathrm{m}]$ & 215 & 150 & 365 & $220 / 190$ & 150 & $107 / 89$ \\
\hline Normalized separation $[\sigma]$ & 9.4 & 12.5 & 12 & 12 & 14 & 15.2 \\
\hline
\end{tabular}

${ }^{\mathrm{a}} 140 \mathrm{~mm}$ aperture with $150 \mathrm{~T} / \mathrm{m}$ gradient in the optics variant SLHC V3.1b.

${ }^{\mathrm{b}}$ Two values indicate two magnet types.

At the aperture bottleneck the free magnet aperture, $r-r_{\text {etc }}$, and the aperture requirements of the beam should be the same, so we can write

$$
\frac{e B_{\mathrm{max}}}{p k} \alpha_{\mathrm{s}}^{2}-r_{\mathrm{etc}}=\left(N_{\mathrm{bsc}}+\frac{N_{\mathrm{bb}}}{2} \sqrt{\alpha_{\mathrm{s}}}\right) \sigma_{x} \alpha_{\mathrm{s}}
$$

We can see that $\alpha_{\mathrm{s}}$ must be

$$
\alpha_{\mathrm{s}}>\sqrt{\frac{p k}{e B_{\max }} \cdot r_{\mathrm{etc}}}
$$

in order to guarantee a free aperture $r>r_{\text {etc }}$. By replacing the beam size at the aperture bottleneck $\sigma_{x}(s)=\sqrt{\epsilon \beta(s)} \approx \sqrt{\frac{\epsilon \cdot m_{12}^{2}}{\beta^{*}}}$, Eq. (20) can be transformed to

$$
\beta^{*}=\frac{\left(N_{\mathrm{bsc}}+\frac{N_{\mathrm{bb}}}{2} \sqrt{\alpha_{\mathrm{s}}}\right)^{2} \cdot \epsilon \cdot m_{12} \cdot \alpha_{\mathrm{s}}^{2}}{\left(\frac{e B_{\max }}{p k} \alpha_{\mathrm{s}}^{2}-r_{\mathrm{etc}}\right)^{2}} .
$$

Note that the non-normalized emittance $\epsilon$ is used, since the energy is constant in this scaling. The matrix element $m_{12}$ can be obtained by inserting the known values of the unscaled lattice. The position of the aperture bottleneck is implicitly given by equating aperture and aperture requirement in Eq. (20) and by assuming the bottleneck will remain at the same (scaled) position in the triplet. The lattice can be scaled, giving the achievable $\beta^{*}$ for any scaling factor. For very large $\alpha_{\mathrm{s}}$, Eq. (22) can be reduced to

$$
\beta^{*} \approx \frac{\frac{N_{\mathrm{bb}}}{2} \epsilon m_{12}}{\frac{e B_{\max }}{p k}} \cdot \frac{1}{\alpha_{\mathrm{s}}},
$$

making any $\beta^{*}$ reachable.

\section{LATTICE OPTIONS}

In the following, the different lattice options for the FCChh interaction region resulting from different scaling strategies will be presented. The different lattice options are named by their $L^{*}$. Important parameters of the different lattices are summarized in Table II. The normalized separation of the counterrotating beams in the shared aperture magnets is expressed in units of beam sizes $\sigma$ in the crossing plane.

\section{A. $L^{*}=46 \mathrm{~m}$}

The first lattice option is obtained from scaling with constant beam stay clear as derived in Sec. II C 1. Applying the corresponding scaling factor $\alpha_{\mathrm{s}}=2$ to the LHC lattice results in an $L^{*}$ of $46 \mathrm{~m}$. The total length of the straight section is $1075.6 \mathrm{~m}$, shorter than the $1.4 \mathrm{~km}$ specified in the preliminary baseline parameter report [3]. The separation section was modified to provide a beam separation of $300 \mathrm{~mm}$. Scaling from the minimum $\beta^{*}=0.4 \mathrm{~m}$ accomplishable in the LHC [14] results in a minimum $\beta^{*}$ of $0.8 \mathrm{~m}$ in the scaled lattice. The corresponding optics are plotted in Fig. 2.

Apart from a thicker beam pipe in Q1, the LHC triplet has no dedicated shielding inside the quadrupoles to protect them from collision debris coming from the IP. In FCC-hh, this is a challenge, as explained in Sec. I.

To study its impact in the final focus triplet, energy deposition simulations were conducted with FLUKA $[15,16]$. This Monte Carlo code is benchmarked up to the TeVenergy region and it is regularly used for beam-machine interaction studies, in particular for the LHC [17] and its high luminosity upgrade [5]. Proton-proton collisions were simulated using the DPMJET-III generator [18,19], directly called from inside FLUKA. The assumed proton-proton inelastic cross section, including inelastic scattering and single diffractive events, is 108 mbarn.

The FLUKA geometry included the TAS absorber in front of the inner triplet, the four superconducting magnets of the triplet, Q1, Q2a, Q2b and Q3, and orbit corrector magnets. In this first study, three orbit correctors were considered: one between Q1 and Q2a, one between Q2a and Q2b and finally one at the end of the triplet. In the other studies reported in this paper, the corrector between Q2a and Q2b was removed.

The cross sections of the quadrupoles and the correctors are shown in Fig. 3. In this first radiation simulation, a coil aperture diameter of $62 \mathrm{~mm}$ was used for all the magnets. 


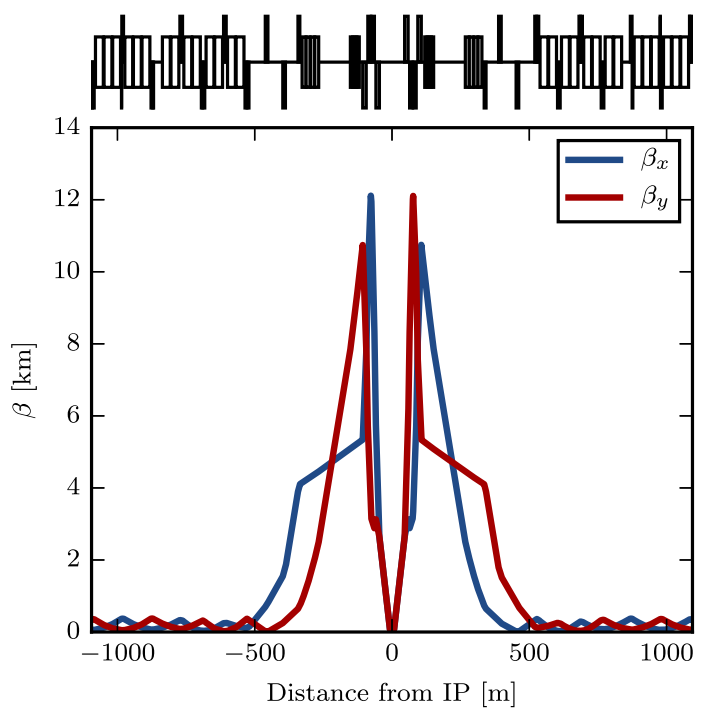

FIG. 2. $\beta$ functions of the scaled LHC lattice with $L^{*}=46 \mathrm{~m}$ for $\beta^{*}=0.8 \mathrm{~m}$. The $\beta$ function in the dispersion suppressors are scaled as well and will be adapted to the arc lattice.

The free aperture radius was further reduced by $0.5 \mathrm{~mm}$ for the Kapton insulator, $1.5 \mathrm{~mm}$ spacing for the liquid helium (LHe) and a $2 \mathrm{~mm}$ thick cold bore, each modeled as a simple layer. The beam screen and clearance were accounted for by additional $2 \mathrm{~mm}$ in the calculation of the beam stay clear, but were not inserted in the FLUKA geometry. The quadrupole cables were assumed to have a thickness of $1.86 \mathrm{~cm}$ and they were included as two layers. A compound material, made of a mixture of $\mathrm{Nb}_{3} \mathrm{Sn}$ and insulator was assumed. A $0.8 \mathrm{~mm}$ thick insulator layer was put to separate them. At that stage, the end cap was modeled as a $9 \mathrm{~cm}$ thick end shoe and a $7.5 \mathrm{~cm}$ thick stainless steel end plate on each side of the quadrupole. The orbit corrector cables were represented as NbTi layers with a thickness of $0.5 \mathrm{~cm}$. The corrector end cap was not included at this stage, causing an overestimate of the energy deposition on the magnet front face. An analytic description of the magnetic field was used for all the magnets. The same magnet model, assuming the same materials and layer thicknesses, was used for all the layouts studied in this paper. The coil aperture was changed, matching the assumed gradient, and the other diameter figures were adjusted accordingly.

The TAS was described as a $3.6 \mathrm{~m}$ long copper cylinder and the drift between the TAS and Q1 was $4.1 \mathrm{~m}$. The TAS aperture diameter was set to $15 \mathrm{~mm}$. The beam pipe between the IP and the TAS has not been modeled at this stage, causing an overestimate of the energy deposition in the TAS, but with no impact on the triplet magnets. With this set of parameters, the triplet is completely in the geometrical shadow of the TAS. The beam stay clear with these parameters is $12 \sigma$ with a beam separation of $12 \sigma$ corresponding to a crossing angle of $86 \mu \mathrm{rad}$.
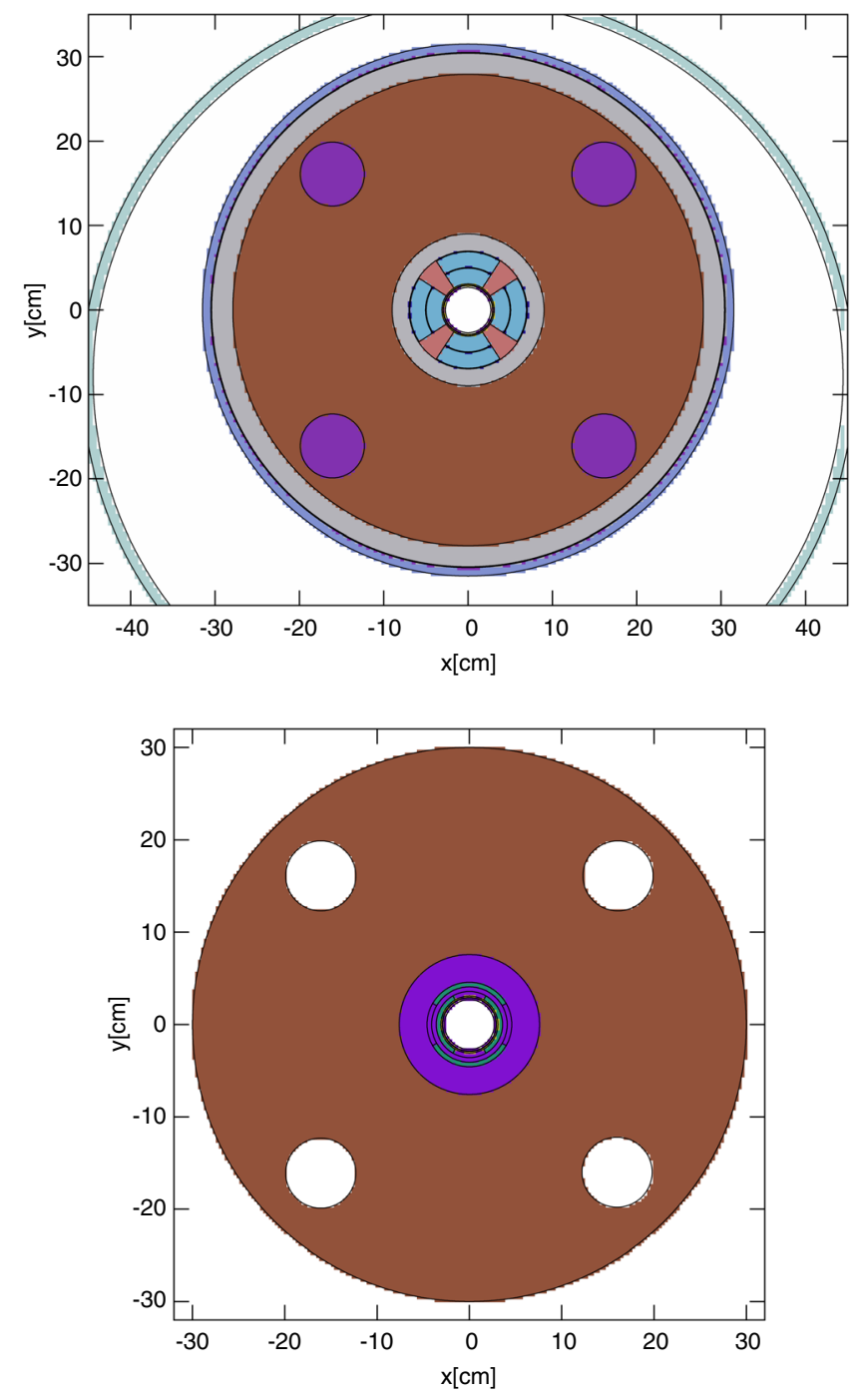

FIG. 3. Magnet cross sections: quadrupole (top) and orbit corrector (bottom).

Figure 4 shows the results of the FLUKA simulation, both in terms of peak power density and peak dose. Both quantities were evaluated averaging on an angular bin of 2 degrees and on a longitudinal bin of $10 \mathrm{~cm}$. For the peak power density the radial average was done on the whole cable dimension, since, in case of a stable thermal configuration in the coil, the relevant quantity for quenches is the average power along the cable. The dose was estimated considering a smaller volume relevant to the insulator damage: the radial bin was reduced to $0.29 \mathrm{~cm}$ for quadrupoles and $0.25 \mathrm{~cm}$ for correctors.

The highest power density occurs at the end of Q1 with about $230 \mathrm{~mW} / \mathrm{cm}^{3}$. This is about 60 times more than the maximum power density in the LHC triplet $\left(4 \mathrm{~mW} / \mathrm{cm}^{3}\right)$. The reference quench limit at $7 \mathrm{TeV}$ is about $40 \mathrm{~mW} / \mathrm{cm}^{3}$ for $\mathrm{Nb}_{3} \mathrm{Sn}$ coils or $13 \mathrm{~mW} / \mathrm{cm}^{3}$ for $\mathrm{NbTi}$ [5]. Looking at the peak dose, the highest values also occur at the end of Q1. With around $2400 \mathrm{MGy}$ per $3000 \mathrm{fb}^{-1}$, the radiation 



FIG. 4. Peak power densities (top) and peak doses (bottom) for the first lattice compared with LHC and HL-LHC for horizontal crossing. The horizontal axes were chosen to overlap similar magnets in the LHC and FCC lattices. The crossing angles were $285 \mu \mathrm{rad}$ for LHC, $590 \mu \mathrm{rad}$ for HL-LHC and $86 \mu \mathrm{rad}$ for FCC.

load is almost 2 orders of magnitude higher than what is currently considered the lifetime limit of 30 MGy [5,20]. It is obvious that these enormous discrepancies between expected load and feasible operational limit, both in terms of peak power and peak dose, need to be addressed. A straightforward option to reduce the radiation load is shielding placed inside the quadrupoles, as it is foreseen for HL-LHC. In the HL-LHC upgrade of LHC, the triplet magnets have an aperture diameter of $150 \mathrm{~mm}$ and shielding of $16 \mathrm{~mm}(6 \mathrm{~mm})$ thickness in Q1 (Q2/Q3). Placing similar amounts in the triplet magnets of the scaled lattice would reduce the free aperture diameter in Q1 to $22 \mathrm{~mm}$ and to $42 \mathrm{~mm}$ in Q2 and Q3. This would greatly limit the reachable $\beta^{*}$ and thereby the instantaneous luminosity of the collider. With the limitation in Q1, not even the baseline goal of $\beta^{*}=1.1 \mathrm{~m}$ would be possible. Furthermore, the same amount of shielding in FCC will be less effective than in HL-LHC due to the higher energy of the debris and the resulting longer penetration depth. The triplet has to be adapted to this challenge. The aperture of the triplet magnets needs to be increased from this first design to allow for the necessary amount of shielding.
Consequently, the gradient must be decreased according to Eq. (10). This can be done by increasing the triplet length to keep the focal length constant or increasing the focal length and thereby $L^{*}$. As both measures also increase the beam size in the triplet, the net gain has to be studied. Either way it is clear that the radiation mitigation measures will be the driving factors of the triplet design.

\section{B. $L^{*}=36 \mathrm{~m}$}

The upscaled LHC lattice only reached a $\beta^{*}$ of $0.8 \mathrm{~m}$ for a beam stay clear of $12 \sigma$ while at the same time leaving no margin for the necessary amounts of shielding. To overcome this problem, the triplet magnet apertures need to be increased. For this purpose, the HL-LHC lattice (specifically the optics variant SLHC V3.1b [5]), having a triplet already $\approx 20 \%$ longer than the LHC, was scaled by the factor 2 according to the scaling derived in Sec. II C 1. After this scaling, the triplet magnets were lengthened by an additional $30 \%$ in order to be able to further decrease the gradients, increase the apertures and subsequently accommodate more shielding. However, a longer triplet results in larger peak $\beta$ functions. To limit this increase, $L^{*}$ was reduced to $36 \mathrm{~m}$ which requires stronger focusing and therefore counteracts the gradient reduction due to the longer triplet. Still a net gain is expected. The $L^{*}$ is close to the upper limit of early detector design studies, suggesting a detector half length between 25 and $40 \mathrm{~m}$ [21]. In order to optimally use the available free aperture, the maximum $\beta$ functions were matched to be the same in both planes, $\beta_{x, \text { max }}=\beta_{y, \text { max }}$.

For the radiation studies, the coil aperture diameter of the triplet magnets was increased to $100 \mathrm{~mm}$. The crossing angle was set to $140 \mu \mathrm{rad}$ corresponding to a beam separation of $12 \sigma$ at $\beta^{*}=0.3 \mathrm{~m}$, a value expected to be feasible from the scaling. The vertical crossing scheme was chosen because it is the worst scenario in terms of total energy deposition [22,23]. The more abundant positive collision debris particles are subject to an earlier capture compared to horizontal crossing, since they enter with a vertical offset in the first quadrupole, where they are further defocused. As explained in the previous section, the free aperture in the FLUKA model was reduced by the cold bore,

TABLE III. Parameters used for the beam stay clear calculation.

\begin{tabular}{lc}
\hline \hline$B_{\max }$ & $11 \mathrm{~T}$ \\
Closed orbit uncertainty & $2 \mathrm{~mm}$ \\
Beta beating & $20 \%$ \\
Layer thickness & \\
Shielding & $15 \mathrm{~mm}$ \\
Liquid helium & $1.5 \mathrm{~mm}$ \\
Kapton insulator & $0.5 \mathrm{~mm}$ \\
Cold bore & $2 \mathrm{~mm}$ \\
Beam screen & $2.05 \mathrm{~mm}$ \\
Beam screen insulation & $2 \mathrm{~mm}$ \\
\hline \hline
\end{tabular}



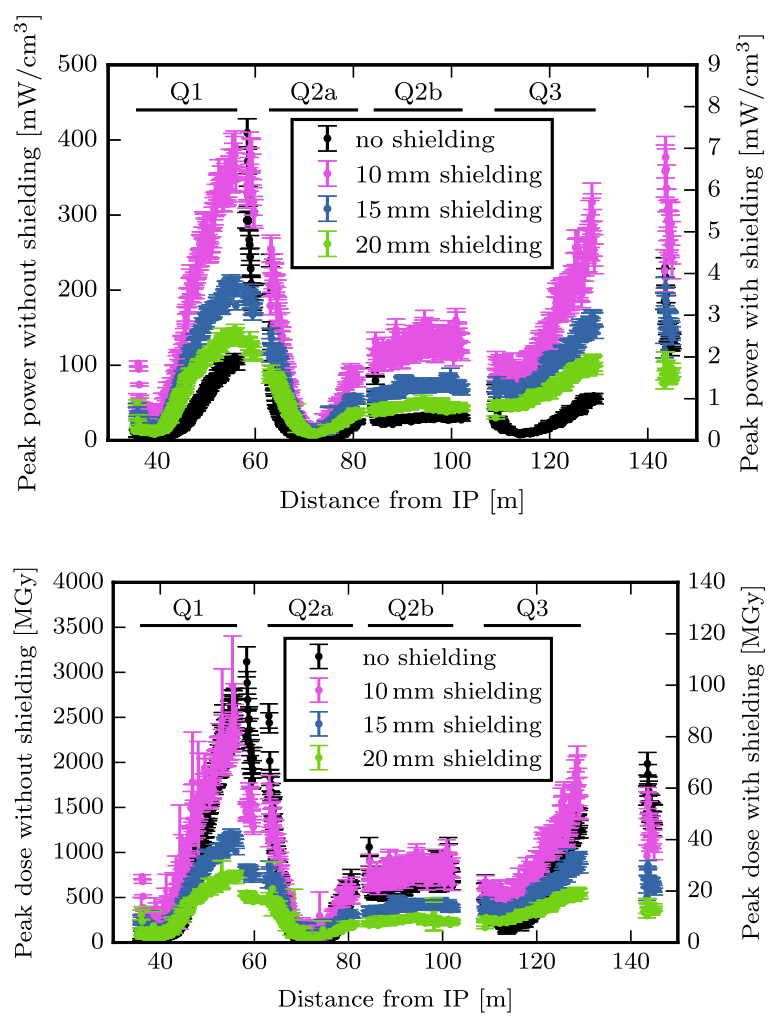

FIG. 5. Peak power densities (top) and peak doses per $3000 \mathrm{fb}^{-1}$ (bottom) for the $L^{*}=36 \mathrm{~m}$ lattice for different shielding thicknesses at $70 \mu \mathrm{rad}$ vertical half crossing angle and assuming $100 \mathrm{~mm}$ coil aperture.

the Kapton insulator and spacing for liquid helium (see Table III) as well as various shielding thicknesses. The shielding was modeled as a continuous layer of INERMET180, a tungsten heavy alloy. In order to leave as much space as possible for the detector, the TAS length was decreased to $3 \mathrm{~m}$ and its distance from Q1 was reduced to $2 \mathrm{~m}$, the assumed minimum space needed for cryostat and vacuum equipment. Its aperture diameter was increased to $20 \mathrm{~mm}$. Figure 5 shows the distributions of the peak power densities and peak doses resulting from the simulation, with different shielding thicknesses ranging from 0 to $20 \mathrm{~mm}$. The results show that, to get a peak dose in the order of $30 \mathrm{MGy}$ for $3000 \mathrm{fb}^{-1}$, a shielding thickness of at least $15 \mathrm{~mm}$ is necessary. For this amount of shielding, the peak power density at the baseline luminosity of $5 \times 10^{34} \mathrm{~cm}^{-2} \mathrm{~s}^{-1}$ remains below $5 \mathrm{~mW} / \mathrm{cm}^{-3}$. Based on the present knowledge, a design limit of the order of $5 \mathrm{~mW} / \mathrm{cm}^{-3}$ is deemed to be reasonably cautious [24]. This first specific figure for the shielding thickness allows to define the available aperture including all required layers and to calculate the beam stay clear. This is necessary to optimize the usage of the available aperture and study the minimum $\beta^{*}$ of the lattice.

To account for the possibility that both peak $\beta_{x, y}$ functions could still be reduced, the beam stay clear itself

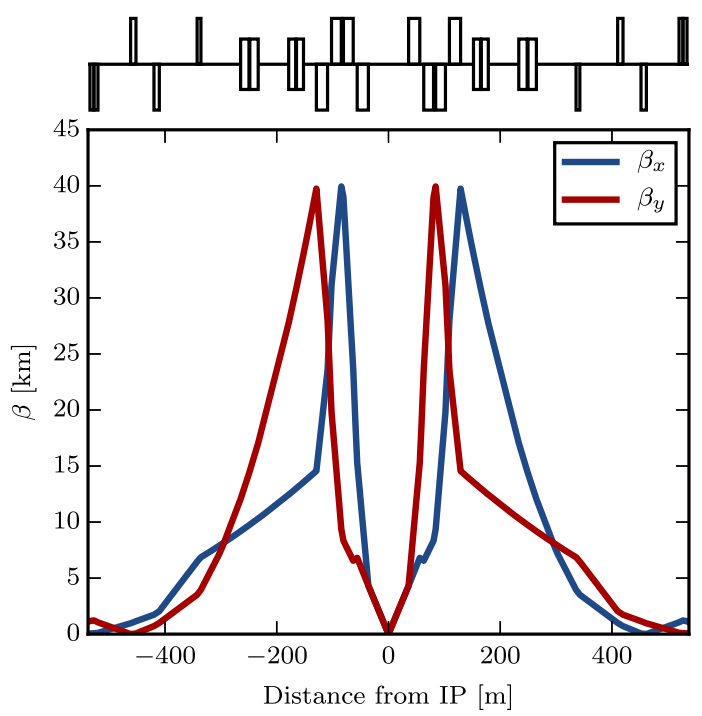

FIG. 6. Interaction region with $L^{*}=36 \mathrm{~m}$ and $\beta^{*}=0.3 \mathrm{~m}$.

was also matched to the required value of e.g. 12 $\sigma$. When this value was met, the matching was repeated with a smaller $\beta^{*}$, until the matching did not converge to a solution anymore, indicating the minimum $\beta^{*}$ possible with this lattice and the given beam stay clear requirement was reached. The beam stay clear for matching was calculated similarly to the APERTURE module of MAD-X [25]: the coil aperture radius was calculated according to Eqs. (9) and (10). From the coil aperture, the closed orbit uncertainty, the shielding thickness and various other layer thicknesses were subtracted (see Table III) to get the available free aperture. This was again divided by the maximum beam size determined by $\beta_{\max }$ to get the beam stay clear.

So far, the quadrupole aperture was assumed to be constant in the whole triplet. The maximum value was defined by the strongest gradient required. When probing the minimum $\beta^{*}$, Q1 happened to be about $20 \%$ stronger than Q2 and Q3. However, the peak $\beta$ functions creating the aperture bottlenecks were located in Q2 and Q3 (see Fig. 6). Their lower gradients allow to increase their coil apertures without exceeding the technical limits, thus increasing the overall beam stay clear. Consequently, a smaller minimum $\beta^{*}$ can be expected when individual apertures are used for Q1 and Q2/Q3.

In Fig. 7 the minimum $\beta^{*}$ as a function of the required beam stay clear is plotted for both uniform apertures (all apertures defined by the strongest gradient in Q1) and for individual apertures (Q2 and Q3 apertures defined by their strongest gradient, Q1 is individual). As expected, the minimum $\beta^{*}$ is considerably better for individual apertures. For uniform apertures, the ultimate goal of $0.3 \mathrm{~m}$ is barely reached. For individual apertures, the minimum $\beta^{*}$ is $0.18 \mathrm{~m}$. Alternatively, the ultimate goal can be reached even for beam stay clear requirements of $17 \sigma$. Furthermore, 


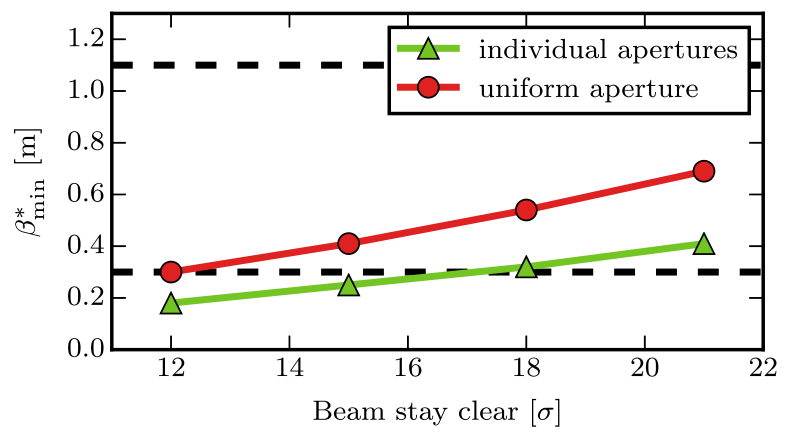

FIG. 7. Minimum $\beta^{*}$ of the $L^{*}=36 \mathrm{~m}$ lattice for beam star clear requirements of $12-21 \sigma$. The baseline goal of $\beta^{*}=1.1 \mathrm{~m}$ poses no problem from the aperture point of view. The "ultimate" goal of $\beta^{*}=0.3 \mathrm{~m}$ can barely be reached with uniform apertures. With individual apertures, the baseline can be reached or exceeded within a beam stay clear of up to $\approx 17 \sigma$.

even with individual apertures, there is still space left in Q1 for more shielding. Up to $24 \mathrm{~mm}$ shielding thickness is possible in Q1 without reducing the overall beam stay clear limited in Q2 and Q3.

The results of the FLUKA simulations with $20 \mathrm{~mm}$ in Fig. 5 can be taken to estimate the gain from more shielding in Q1. It should be noted that in the model used in FLUKA, the shielding thickness is kept constant along the triplet, although $20 \mathrm{~mm}$ of shielding in Q2 and Q3 would limit the beam stay clear. To get a realistic estimate, the peak doses for $20 \mathrm{~mm}$ in Q1 and for $15 \mathrm{~mm}$ from Q2 onwards should be regarded. With this in mind, the maximum peak dose for $3000 \mathrm{fb}^{-1}$ is in Q3 and reaches about $30 \mathrm{MGy}$, a value that is considered feasible with current technology. In case of individual apertures, we can expect the peak doses at the beginning of Q2 and the end of Q3 to drop as the coils are more retracted and exposed to less radiation. With individual apertures, the maximum peak dose will thus be at the end of Q1 with about 27 MGy. In this case the magnets should be able to withstand the integrated luminosity of $3000 \mathrm{fb}^{-1}$. However, for ultimate parameters, one high luminosity run is expected to average $1000 \mathrm{fb}^{-1}$ /year over a five-year period [3]. Thus the magnets must be able to withstand an integrated luminosity of at least $5000 \mathrm{fb}^{-1}$ to allow running the full period without replacing them. Studies of irradiation of the LHC triplet after only $300 \mathrm{fb}^{-1}$ (at $L=1 \times 10^{34} \mathrm{~cm}^{-2} \mathrm{~s}^{-1}$ ) estimate a 4 to 6 months cool-down time before the ambient doses in the triplet area are low enough to allow work on the magnet exchange [26]. With $5000 \mathrm{fb}^{-1}$ per operational cycle and an instantaneous luminosity of $L=5 \times 10^{34} \mathrm{~cm}^{-2} \mathrm{~s}^{-1}$ the necessary cool-down times will become considerably longer for FCC-hh. Together with 6 to 8 months of work required for the magnet exchange, this becomes incompatible with the foreseen shut-down time of 1.5 years as for the LHC [3]. Therefore, it is desirable to survive an integrated luminosity equivalent to several high luminosity

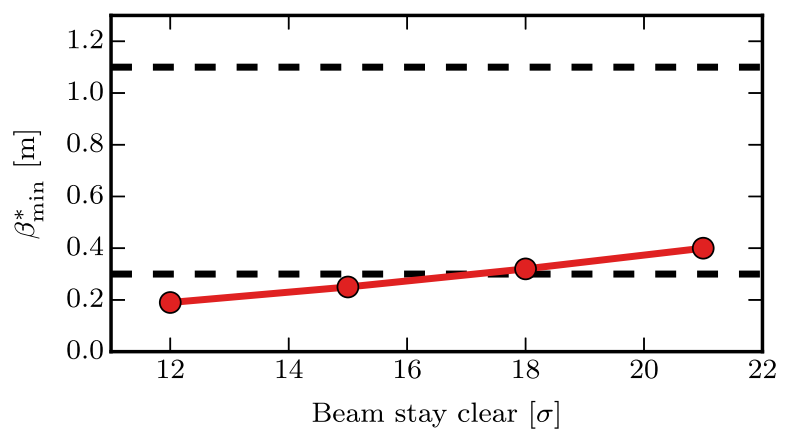

FIG. 8. $\quad \beta^{*}$ reach of the $L^{*}=61 \mathrm{~m}$ lattice for beam stay clear requirements of $12-21 \sigma$. The reachable $\beta^{*}$ is very similar to the one of the $L^{*}=36 \mathrm{~m}$ lattice with individual apertures. Note that the triplet magnets in the $L^{*}=61 \mathrm{~m}$ all have similar gradients, so all apertures are the same.

runs or even the whole foreseen scenario of $17500 \mathrm{fb}^{-1}$. To achieve this goal, the triplet needs further optimization to reduce the radiation load, preferably without concessions in performance.

\section{C. $L^{*}=61 \mathrm{~m}$}

Applying the scaling factor $\alpha_{\mathrm{s}}=\sqrt{\frac{50 \mathrm{TeV}}{7 \mathrm{TeV}}} \approx 2.67$ for constant gradients and apertures, as discussed in Sec. IIC 2, to the HL-LHC lattice (version SHLC V3.1b) leads to $L^{*}=61.5 \mathrm{~m}$. Due to the increased distance between IP and separation dipoles, the normalized separation had to be increased to $14 \sigma$ in order to keep the effect of long range beam-beam interactions constant.

The $\beta^{*}$ reach of the new lattice was calculated as described previously. The scaling with constant gradient $\partial B_{y} / \partial x=150 \mathrm{~T} / \mathrm{m}$ and constant apertures $2 r=140 \mathrm{~mm}$ corresponds to $B_{\max }=10.5 \mathrm{~T}$. The resulting $\beta_{\min }^{*}$ are plotted in Fig. 7. Unlike in the $L^{*}=36 \mathrm{~m}$ lattice, the matching of the triplet at $L^{*}=61 \mathrm{~m}$ for a maximum beam stay clear resulted in very similar quadrupole gradients for the whole triplet, so an option with individual apertures offers minor advantages. The comparison between Figs. 8 and 7 reveals that the minimum $\beta^{*}$ reachable in both lattices is the same. Although the relative impact of the shielding on the free aperture is smaller due to a larger coil aperture, the increased normalized separation increases the orbit excursion and thereby reduces the beam stay clear. The maximum $\beta$ functions in the triplet and the associated expected gain in $\beta^{*}$ min are thereby limited. The different $B_{\max }$ only has a small impact.

Although the scaling leading to $L^{*}=61 \mathrm{~m}$ did not improve the minimum $\beta^{*}$, it is worth studying the impact on the energy deposition. Figure 9 shows radiation load for the new lattice alongside the load for the $L^{*}=36 \mathrm{~m}$ lattice. The peak doses in the most problematic areas were reduced by about $20 \%$ at constant shielding thickness. The main reasons for this are the larger coil apertures as well as the reduced gradients, limiting the particle losses in the 


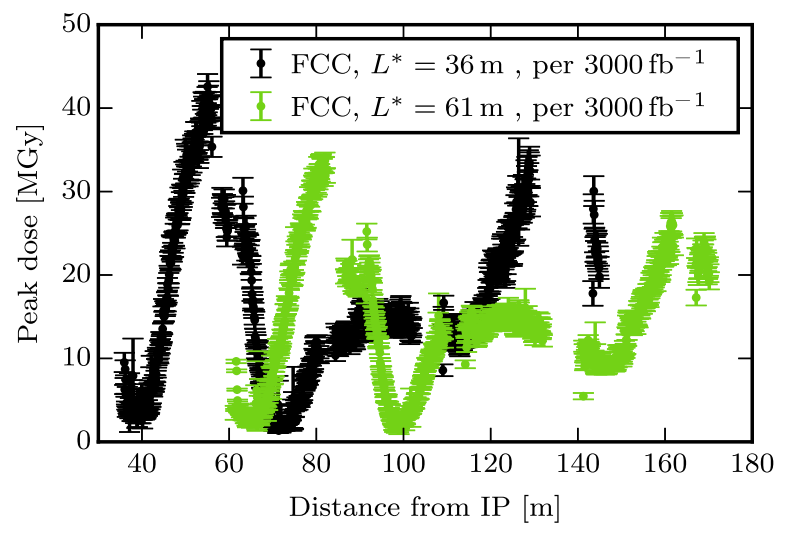

FIG. 9. Comparison of the peak doses per $3000 \mathrm{fb}^{-1}$ for the $L^{*}=36 \mathrm{~m}$ and the $L^{*}=61 \mathrm{~m}$ lattices with $15 \mathrm{~mm}$ shielding.

defocusing planes. A counteracting effect comes from the larger crossing angle but the overall effect is a reduced load.

\section{D. $L^{*}=45 \mathrm{~m}$}

Although $L^{*}$ increased significantly with $\alpha_{\mathrm{s}}=\sqrt{\frac{50 \mathrm{TeV}}{7 \mathrm{TeV}}}$ in the previous case, the lengths of the triplet magnets are almost the same as in the $L^{*}=36 \mathrm{~m}$ lattice due to the $30 \%$ length increase performed on the $L^{*}=36 \mathrm{~m}$ lattice. The impact of changing the triplet length versus changing $L^{*}$ is therefore worth studying. However, changing the ratio of the triplet lengths and $L^{*}$ will require rematching in order to have optimized optics, making analytical descriptions of the beam stay clear of the optimized lattice nontrivial. Instead the scaling length scaling approach to minimize $\beta^{*}$ deduced in Sec. II C 3 can be used to qualitatively describe $\beta^{*}$ reach vs $L^{*}$ and triplet length in the presence of considerable amounts of shielding.

For this purpose, the scaling of $\beta^{*}$ described in Eq. (22) is applied to the lattices studied above. Figure 10 shows the resulting $\beta^{*}$ reach when the formula is applied to the $L^{*}=$ 36 m lattice (top) and the $L^{*}=61 \mathrm{~m}$ lattice (bottom). The markers show the cases where the analytically scaled $\beta^{*}$ reach was verified by MAD-X aperture calculations. The region with $\alpha_{\mathrm{s}}>1$ is of most interest as $\beta_{\min }^{*}$ decreases with $1 / \alpha_{\mathrm{s}}$. In this region, the plots show that both scaled lattices feature almost the same minimum $\beta^{*}$ at the same scaling factors. Furthermore, both lattices have almost the same lengths of the triplet magnets for any scaling factor (difference $2.5 \%$ ). Consequently, only $L^{*}$ is significantly differing with the scaling factor. This suggests that $L^{*}$ alone has a rather small impact. The main contribution for the smaller minimum $\beta^{*}$ at larger scaling factors seems to be caused by the triplet length as can be seen by comparing the $\beta^{*}$ reach in both scalings at equal $L^{*}$. For example, for an $L^{*}$ of $60 \mathrm{~m}$ the minimum $\beta^{*}$ at $12 \sigma$ beam stay clear is $0.04 \mathrm{~m}$ in the upper plot of Fig. 10 but only $0.21 \mathrm{~m}$ in the lower plot. The significant differences here are the lengths of the triplet magnets.
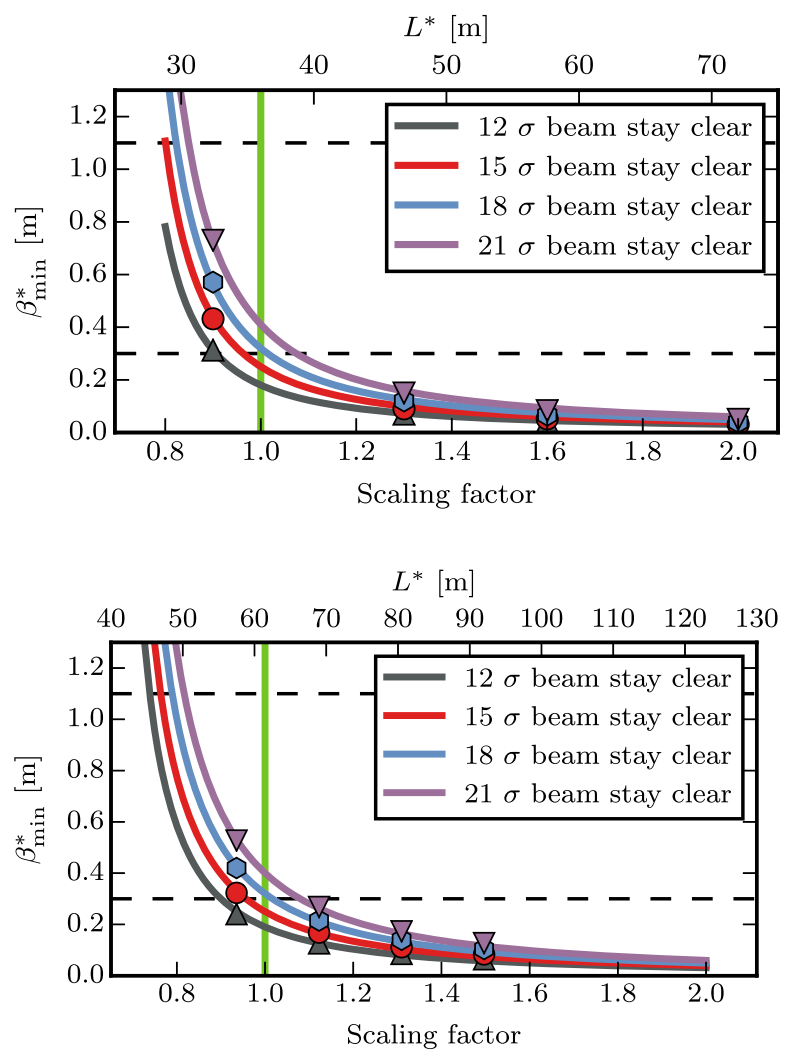

FIG. 10. Minimum $\beta^{*}$ for the $L^{*}=36 \mathrm{~m}$ lattice (top) and $L^{*}=$ $61.5 \mathrm{~m}$ (bottom) lattice analytically scaled in length, scaling factor and resulting $L^{*}$ (top horizontal axis). Markers show $\beta_{\text {min }}^{*}$ values obtained with MAD-X aperture calculations. The green vertical lines indicate the nonscaled value.

These observations suggest a clear strategy to maximize the $\beta^{*}$ reach with significant amounts of shielding reducing the free aperture of the final focus magnets: to choose the smallest $L^{*}$ that does not restrict the detector design and to increase triplet length until dynamic aperture or chromaticity become obstacles.

Following this design strategy as well as new requirements from the detector baseline design [27], the $L^{*}=$ $45 \mathrm{~m}$ lattice was developed. A feature of the new detector design is the introduction of a horizontal forward spectrometer dipole with an integrated field of $10 \mathrm{Tm}$ that must be compensated by a corrector dipole.

Figure 11 shows a sketch of one side of the detector layout. The cavern ends at $z=35 \mathrm{~m}$. The space of $3 \mathrm{~m}$ between the end of the forward muon spectrometer and tunnel is needed to open up the detector and cannot be filled with immovable equipment. The forward spectrometer is located at a distance of 14.8 to $21 \mathrm{~m}$ from the IP. For the compensator dipole at $z=33 \mathrm{~m}$ normal conducting technology was chosen due to its robustness in the highly radiative environment [28]. In addition, no cryostat is needed, easing the removal of the magnet for detector maintenance. Placed before the shielding wall, the orbit corrector must not be hit by physics debris, in order to 


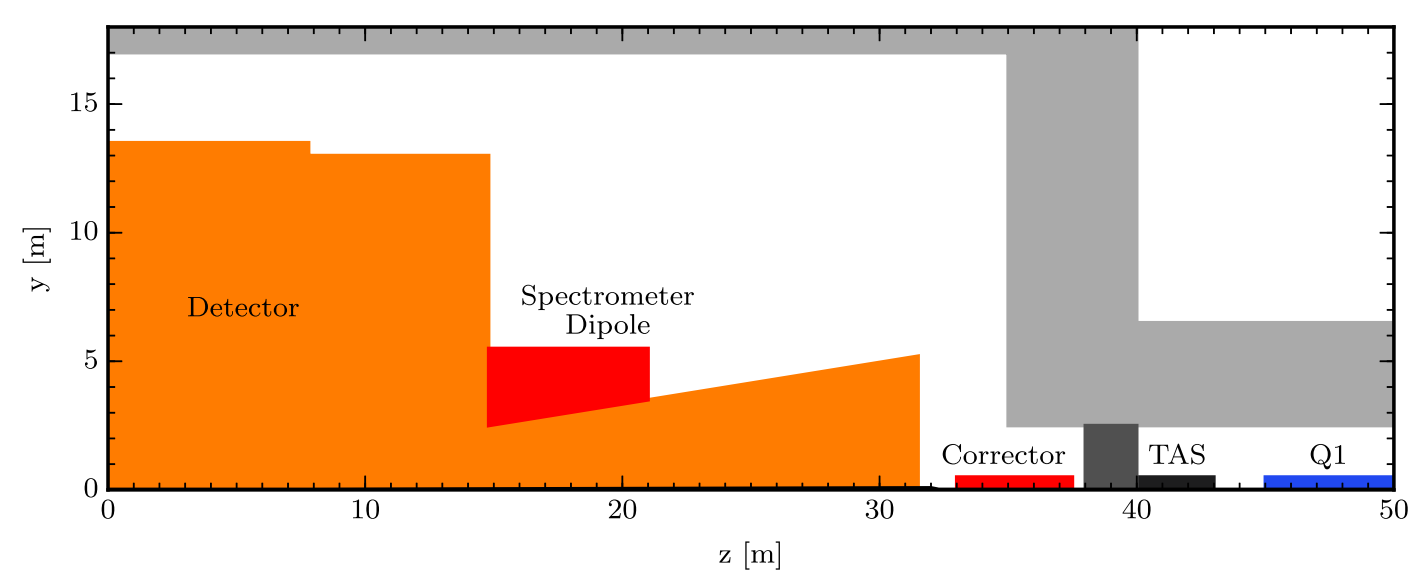

FIG. 11. Detector and interaction region layout leading to the $L^{*}=45 \mathrm{~m}$ lattice. The IP is located at $(0,0)$.

avoid background in the detector. Thus, a large aperture is required. Hadrons hitting the TAS create secondary particles that the detector needs to be shielded from. In the LHC, ATLAS is protected by the two forward shielding assemblies, each consisting of more than 400 tonnes of iron and steel on a bridge section as well as a nose shielding of 117 tonnes of iron per side. CMS is protected by partly movable forward shielding weighting about 260 tonnes per side in addition to support structures of the similar mass. To avoid mechanical challenges and limit costs, the TAS of FCC-hh is supposed to be housed in the tunnel, behind a shielding wall of $2 \mathrm{~m}$ thickness. Taking the lengths of the detector, the corrector dipole, TAS and shielding wall together and allowing for some margin for connections and vacuum equipment, a minimum $L^{*}$ of $45 \mathrm{~m}$ is considered realistic.

In order to reduce the minimum $\beta^{*}$, the triplet magnets were lengthened by $50 \%$ to a length of $31 \mathrm{~m}$ for Q1 and Q3 and $26 \mathrm{~m}$ for Q2a and Q2b.

The crossing angle is determined by the number of long range beam-beam interactions between IP and separation dipole. Increasing the distance traveled in a shared aperture, i.e. increasing the number of long range interactions, must be compensated by a larger normalized separation. Applying the scaling factor according to Eq. (19) yields a normalized separation of $15.2 \sigma$. For the ultimate parameters with $\beta^{*}=0.3 \mathrm{~m}$, this corresponds to a half crossing angle of $89 \mu \mathrm{rad}$. This value needs to be adapted to the spectrometer and orbit corrector since they interfere with the separation. For a beam energy of $50 \mathrm{TeV}$, the
$10 \mathrm{Tm}$ of the spectrometer corresponds to a bending angle of $60 \mu \mathrm{rad}$, the $-7 \mathrm{Tm}$ orbit corrector to $-42 \mu \mathrm{rad}$, leaving an additional angle of $60 \mu \mathrm{rad}-42 \mu \mathrm{rad}=18 \mu \mathrm{rad}$, the "experimental angle," to be added or subtracted to the internal crossing angle. The resulting external crossing angle will either result in an unnecessarily large aperture need or in an increased integrated long range beam-beam effect. To avoid these consequences, the internal crossing angle (i.e. the crossing angle at the IP) needs to be scaled to keep the beam-beam effect constant. Crossing schemes with similar beam-beam effect are shown in Table IV.

The considerable increase of the triplet magnet lengths led to aperture diameters of $205 \mathrm{~mm}$ in Q1 and $248 \mathrm{~mm}$ in Q2 and Q3. The option to use individual apertures was used to maximize the $\beta^{*}$ reach as we have already shown in Sec. III B. A shielding of $15 \mathrm{~mm}$ was assumed all along the triplet. For a more realistic design, tentative gaps were inserted in the interconnects. As expected from the scaling studies, the increase of triplet magnet lengths by $50 \%$ increased the $\beta^{*}$ reach: Fig. 12 shows the available beam stay clear for various ambitious $\beta^{*}$ settings. When accepting a minimum beam stay clear of $12 \sigma, \beta^{*}$ can be pushed down to $0.05 \mathrm{~m}$. This corresponds to the lowest $\beta^{*}$ configuration with advantages for the luminosity production rate [29]. Alternatively, for the ultimate $\beta^{*}=0.3 \mathrm{~m}$, the beam stay clear reaches $42 \sigma$, a value much larger than necessary for collimation. The free aperture can be used for additional shielding to reduce the radiation load further. As stated above, the ideal goal is to survive an integrated luminosity of $17500 \mathrm{fb}^{-1}$.

TABLE IV. Crossing schemes with similar long range beam-beam effect.

\begin{tabular}{lcc}
\hline \hline Internal half crossing angle & Experimental angle & External angle \\
\hline $110 \mu \mathrm{rad}$ & $-18 \mu \mathrm{rad}$ & $92 \mu \mathrm{rad}$ \\
$89 \mu \mathrm{rad}$ & $0 \mu \mathrm{rad}$ & $89 \mu \mathrm{rad}$ \\
$71 \mu \mathrm{rad}$ & $18 \mu \mathrm{rad}$ & $89 \mu \mathrm{rad}$ \\
$85 \mu \mathrm{rad}$ (vertical) & $\pm 18 \mu \mathrm{rad}$ & $85 \mu \mathrm{rad}(\mathrm{vertical}) \pm 18 \mu \mathrm{rad}($ horizontal $)$ \\
\hline \hline
\end{tabular}




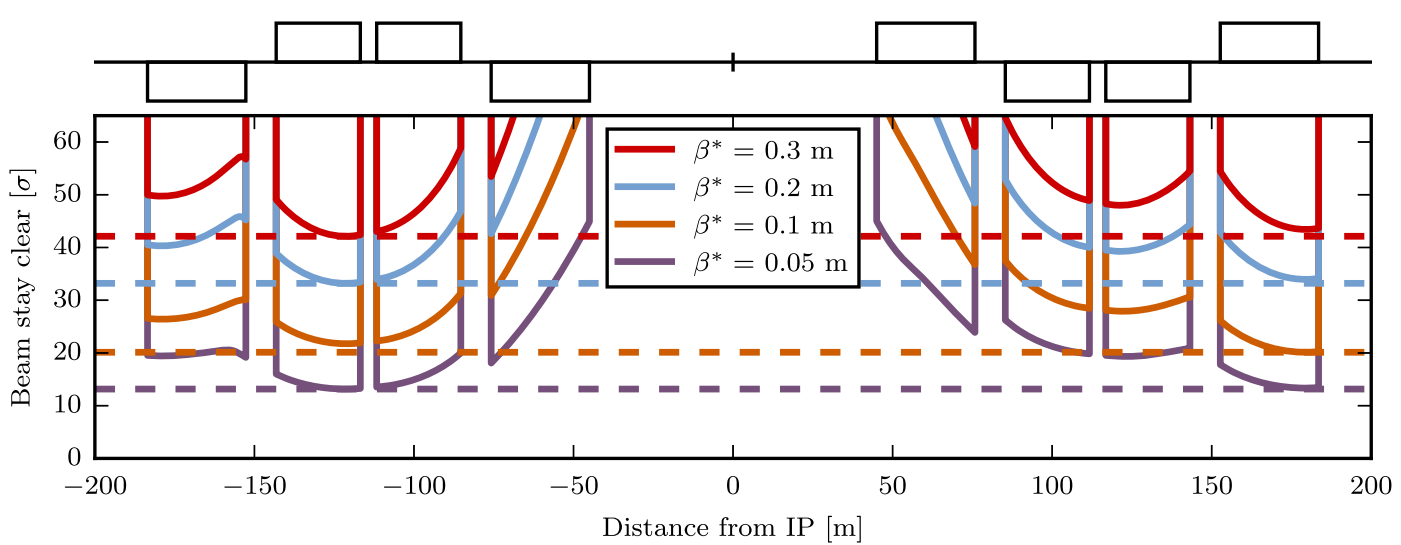

FIG. 12. Beam stay clear of the $L^{*}=45 \mathrm{~m}$ lattice for different values of $\beta^{*}$. Allowing a beam stay clear of only $12 \sigma$, the aperture allows a $\beta^{*}$ down to $0.05 \mathrm{~m}$. Alternatively, the ultimate baseline goal of $\beta^{*}=0.3 \mathrm{~m}$ leaves a beam stay clear of $42 \sigma$, allowing thicker shielding. The beam stay clear was calculated with $15 \mathrm{~mm}$ of shielding in every magnet.

The radiation load in the new lattice will be influenced by the spectrometer dipole. In order to compare the $L^{*}=$ $45 \mathrm{~m}$ scenario with the previous lattices, the spectrometer was switched off for the first studies. The upper plot in Fig. 13 shows the resulting peak doses for horizontal crossing, the lower plot for vertical crossing. Comparing the maximum at the end of Q1 with the previous results in
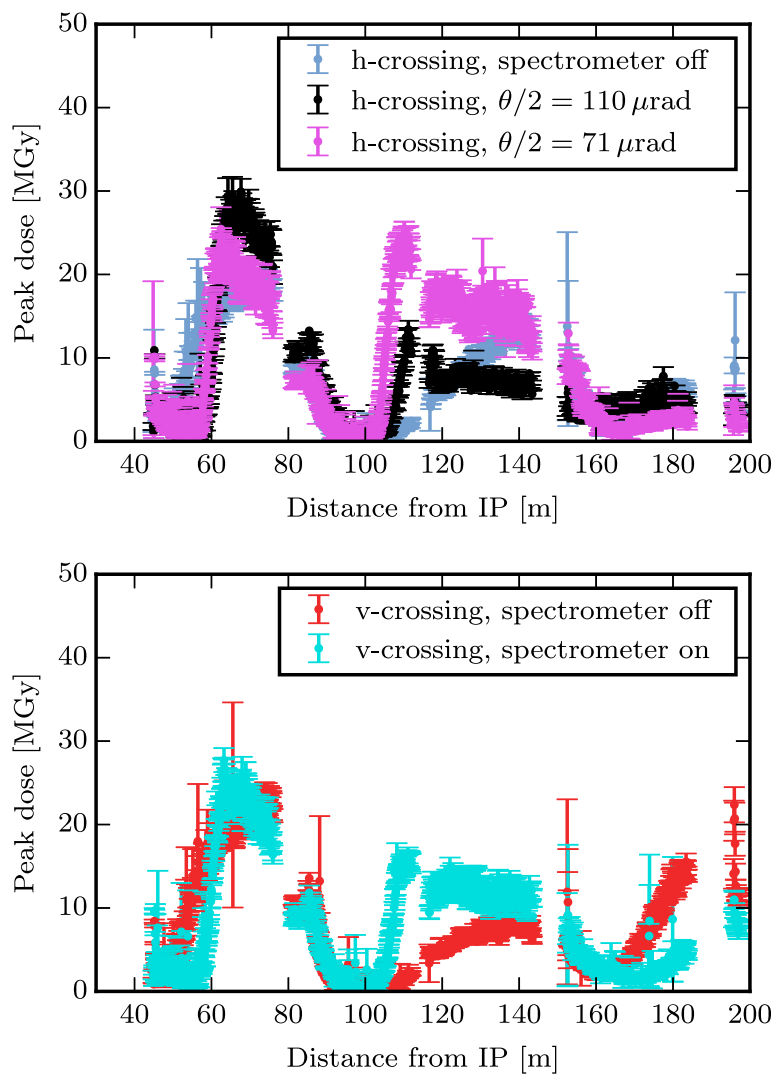

FIG. 13. Peak dose distributions per $3000 \mathrm{fb}^{-1}$ for the $L^{*}=$ $45 \mathrm{~m}$ scenario with and without spectrometer. The strong early deflection of the debris due to the spectrometer leads to a significant peak dose increase, most notably in Q1.
Fig. 9 shows a reduction of the peak dose by $44 \%$ with respect to the $L^{*}=36 \mathrm{~m}$ lattice and $26 \%$ with respect to the $L^{*}=61 \mathrm{~m}$ lattice. This is again caused by a larger coil aperture and a lower gradient. With a peak dose of $20 \mathrm{MGy}$ per $3000 \mathrm{fb}^{-1}$ for horizontal crossing, this is close to the minimum goal of 30 MGy per $5000 \mathrm{fb}^{-1}$.

The plots also show the dose distributions with the experimental spectrometer and its compensator switched on. The first case corresponds to an internal half crossing angle of $110 \mu \mathrm{rad}$ and an experimental angle of $-18 \mu \mathrm{rad}$ (see Table IV). This was considered the worst case scenario in terms of dose, because here debris particles have the largest initial transverse momentum and largest offset when entering Q1.

As expected, the load in Q1 increased significantly. Apart from the larger crossing angle, this is mainly due to the increased capture of negative pions that are deflected further outwards by the combined effects of spectrometer and corrector dipole, as well as by the field in Q1 [30]. The inverted spectrometer option for horizontal crossing corresponds to the internal half crossing angle of $71 \mu \mathrm{rad}$ and $18 \mu \mathrm{rad}$ experimental angle. As expected, the load in the triplet is smaller for this spectrometer orientation as the initial horizontal offset of the debris is smaller. For the vertical crossing, the orientation of the horizontal spectrometer has no impact on the longitudinal dose distribution. However, the azimuthal distribution of the load is affected significantly, opening up opportunities for crossing angle gymnastics for radiation mitigation as described in [30].

As an exploratory study towards a sustainable integrated luminosity of $17500 \mathrm{fb}^{-1}$, the shielding inside the triplet magnets was increased to $55 \mathrm{~mm}$ thickness. With this amount of shielding the beam stay clear reduced to $15.5 \sigma$ at a minimum $\beta^{*}$ of $0.2 \mathrm{~m}$. While this is still lower than the ultimate parameter, it considerably limits the luminosity that the long triplet could offer beyond that. The resulting peak doses are plotted in Fig. 14. Compared to the case with 


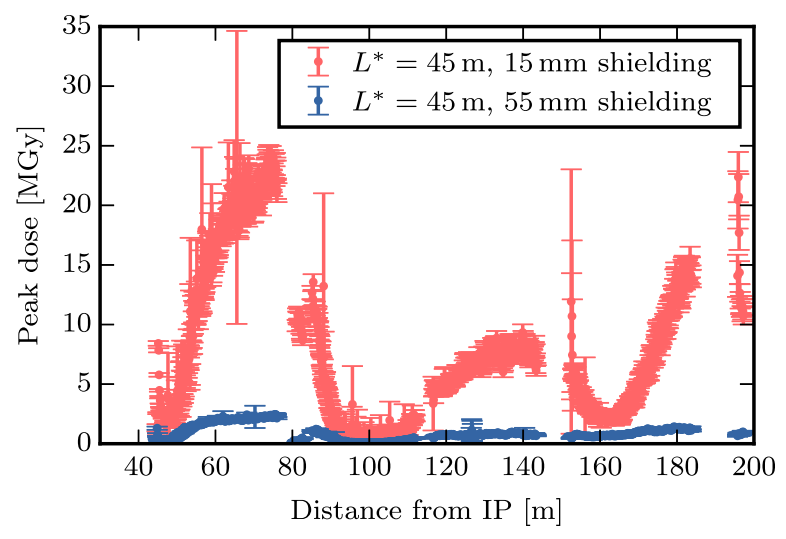

FIG. 14. Peak dose distributions $L^{*}=45 \mathrm{~m}$ for vertical crossing with a shielding thickness of $55 \mathrm{~mm}$ for an integrated luminosity of $3000 \mathrm{fb}^{-1}$. The massive increase in shielding reduced the dose by a factor of 10 .

$15 \mathrm{~mm}$ of shielding, the maximum peak dose was reduced by a factor of 10 . This means, if the available aperture offered by the length increase is used for shielding, the triplet could already survive the full integrated lifetime luminosity while offering some limited margin in $\beta^{*}$ or beam stay clear. The thick shielding also reduced the peak power density below $2 \mathrm{~mW} / \mathrm{cm}^{3}$ for the ultimate luminosity of $30 \times 10^{34} \mathrm{~cm}^{-2} \mathrm{~s}^{-1}$. This value offers a comfortable margin to the design limit of $5 \mathrm{~mW} / \mathrm{cm}^{3}$.

\section{RADIATION MITIGATION BY SPLITTING Q1}

Apart from the $L^{*}=45 \mathrm{~m}$ option with very thick shielding, all lattices presented so far exceed the minimum goal of a maximum peak dose of $30 \mathrm{MGy}$ per operational cycle of $5000 \mathrm{fb}^{-1}$. As discussed above, the expected cooldown times for the irradiated magnets make the survival of the whole FCC-hh lifetime (equivalent to $17500 \mathrm{fb}^{-1}$ ) desirable. Various methods have been proposed, including more radiation resistant materials in the magnets as well as optimized running scenarios in which the radiation is distributed over different areas. As the highest peak dose has always appeared around the end of Q1, another option is to distribute the radiation load more evenly over the length of the magnet. This can be done by splitting Q1 into two different quadrupoles with individual apertures and subsequently individual gradients. Due to the parametrization presented in this section, the feedback of this method on the beam optics is minimized. Unlike a simple increase of the shielding thickness, the beam stay clear is not reduced by the Q1 split. The basic principle of this method makes it applicable independently of $L^{*}$ and triplet length. We first introduced the method in [31].

\section{A. Method and parametrization}

The goal of splitting Q1 is to reduce the radiation load in the first triplet magnet with minor impact on the optics.
Therefore, the total integrated quadrupole strength will be kept constant.

$$
\int_{\mathrm{Q} 1} k \mathrm{~d} s=\text { const. }
$$

Furthermore, the length $L$ of Q1 is kept constant. In this way, the geometry remains constant and the change of the $\beta$ functions in the triplet will be kept minimal. The lengths of Q1a and Q1b are defined by the ratio $\lambda$ :

$$
L_{\mathrm{Q} 1 \mathrm{a}}=\lambda \cdot L_{\mathrm{Q} 1 \mathrm{~b}}
$$

Since $L_{\mathrm{Q} 1 \mathrm{a}}+L_{\mathrm{Q} 1 \mathrm{~b}}=L$, we can deduce

$$
\begin{gathered}
L_{\mathrm{Q} 1 \mathrm{a}}=L \cdot \frac{\lambda}{1+\lambda} \\
L_{\mathrm{Q} 1 \mathrm{~b}}=\frac{L}{1+\lambda} .
\end{gathered}
$$

In order to have different apertures, the gradients must be different. We introduce the ratio of the gradients $r_{\mathrm{g}}$ with

$$
k_{\mathrm{Q} 1 \mathrm{a}}=r_{\mathrm{g}} \cdot k_{\mathrm{Q} 1 \mathrm{~b}},
$$

so the gradient of Q1a is $r_{\mathrm{g}}$ times stronger than the gradient of Q1b. For the goal of reducing the radiation load in Q1b, $r_{\mathrm{g}}$ will be larger than 1 . Since the integrated strength should be constant, we can deduce

$$
k_{\mathrm{Q} 1 \mathrm{a}} \cdot L_{\mathrm{Q} 1 \mathrm{a}}+k_{\mathrm{Q} 1 \mathrm{~b}} \cdot L_{\mathrm{Q} 1 \mathrm{~b}}=k \cdot L .
$$

Inserting Eqs. (25) and (28) in (29) yields

$$
\begin{gathered}
k_{\mathrm{Q} 1 \mathrm{a}}=k \frac{1+\lambda}{\frac{1}{r_{\mathrm{g}}}+\lambda} \\
k_{\mathrm{Q} 1 \mathrm{~b}}=k \frac{1+\lambda}{1+r_{\mathrm{g}} \lambda} .
\end{gathered}
$$

Thus, in a given lattice with given $k$ and $L, 2$ degrees of freedom remain for the radiation load minimization: $r_{\mathrm{g}}$ and $\lambda$. For optics adjustments, the parameter $k$ is used.

In Fig. 15, the variations of the $\beta$ functions for different values of $r_{\mathrm{g}}$ and $\lambda$ are shown. As intended, they do not change remarkably within the triplet, even without rematching.

Again we use the aperture-gradient model of Eq. (18). For this study, $B_{\max }$ was set to $11 \mathrm{~T}$. Inserting Eqs. (30) and (31) into Eq. (18) yields

$$
r_{\mathrm{Q} 1 \mathrm{a}}=r_{\mathrm{Q} 1} \frac{\frac{1}{r_{\mathrm{g}}}+\lambda}{1+\lambda}
$$




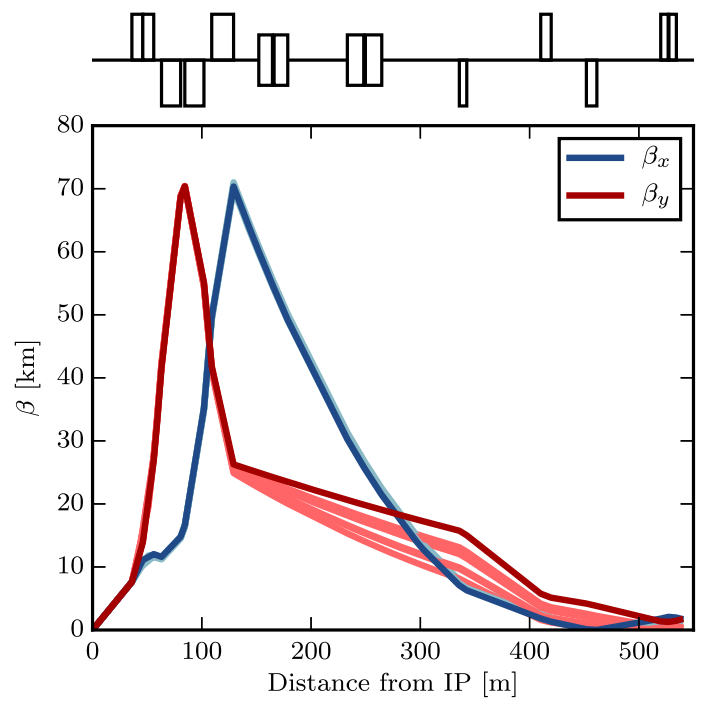

FIG. 15. Change of the optics from unsplit (dark) to split (light) Q1 for $r_{\mathrm{g}}=1.2$ and 1.5 , each with $\lambda=0.3,1.0,3.0$. No rematching was performed.

$$
r_{\mathrm{Q} 1 \mathrm{~b}}=r_{\mathrm{Q} 1} \frac{1+r_{\mathrm{g}} \lambda}{1+\lambda}
$$

where $r_{\mathrm{Q} 1}$ is the coil aperture of the unsplit Q1. For the goal of reducing the radiation load in $\mathrm{Q} 1 \mathrm{~b}, r_{\mathrm{g}}$ will be larger than 1 as this decreases the gradient of Q1b and allows for a larger aperture. To minimize the radiation load, it is better to insert as much shielding as possible without reducing the minimum beam stay clear. On the assumption that the beam size only changes negligibly, an increase in coil aperture in Q1b allows to increase the shielding thickness by the same amount. Similarly, in Q1a the shielding has to be reduced when the coil aperture is shrinking. It should be noted that in the unsplit case, the shielding thickness is constant along Q1 and determined by the beam stay clear at the end. This means, at the entrance of Q1, the shielding is not maximal. For the Q1 split, Q1a will receive larger doses, so the shielding will be chosen as thick as possible. Consequently, when directly comparing the split and the unsplit case, the shielding thickness of Q1a can be larger.

\section{B. Effects of splitting Q1}

In Fig. 16 the peak dose of the unsplit Q1 is illustrated by a black line. The dotted red and green lines qualitatively show the expected dose for a split Q1: for a stronger gradient in Q1a, the coil aperture will become smaller, thus increasing the dose. Since the gradient is larger, debris particles are defocused stronger than before, giving an additional effect on the radiation load that increases with the distance from the IP. Due to the smaller coil aperture, the shielding thickness that can be placed in Q1a will become smaller when increasing $r_{\mathrm{g}}$, resulting in an even larger radiation load in the magnet coils. However, as

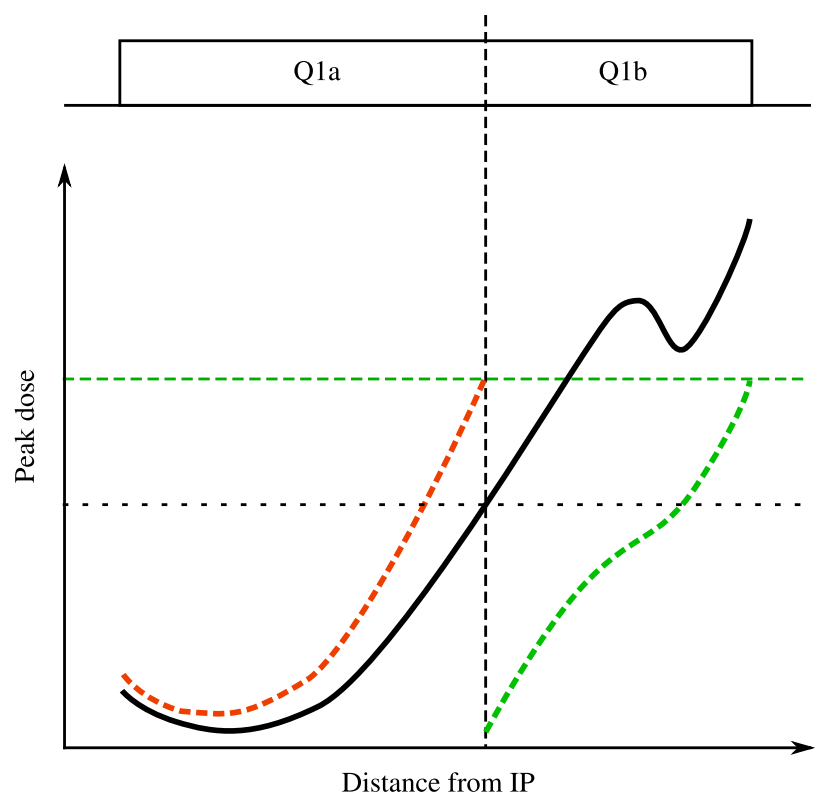

FIG. 16. Qualitative sketch of the peak dose in the Q1. The horizontal axis extends over the length of $\mathrm{Q} 1(\mathrm{a} / \mathrm{b})$ shown at the top. The black line describes the peak dose for an unsplit Q1. The expected changes for splitting Q1 are shown by the dashed red and green lines.

discussed above, the shielding could in principle be thicker closer to the IP compared to the unsplit case. Placing the most possible shielding in Q1a may therefore counteract the increased radiation dose at first.

In Q1b the gradient is decreased, giving a larger possible coil aperture. The retracted coils will be exposed to less radiation. The entrance of $\mathrm{Q} 1 \mathrm{~b}$ should be completely protected by the shielding in Q1a while the exit is more exposed to debris particles deflected outwards by the quadrupole field. Since the beam size is intended to be kept roughly the same as in the unsplit Q1, the larger coil aperture also leaves space for thicker shielding, decreasing the dose further.

\section{Effects of the free parameters}

First, the effect of the gradient ratio $r_{\mathrm{g}}$ on the radiation load will be studied. For $r_{\mathrm{g}}=1$ we have the initial situation of an unsplit Q1. A lower ratio is undesirable since it will reduce the aperture at the end of Q1, the point that already features the highest radiation load. As discussed before, increasing $r_{\mathrm{g}}$ will increase the peak dose in Q1a and reduce the one in Q1b. The optimum $r_{\mathrm{g}}$ is reached at the point where the highest doses in both magnets are the same, meaning both magnets can sustain the same integrated luminosity (green dotted horizontal line in Fig. 16).

As discussed earlier, the radiation load in Q1a can only increase. Thus, the load at the end of Q1a for $r_{\mathrm{g}}=1$ (black dotted horizontal line in Fig. 16) is a lower limit for the achievable maximum peak dose. From this point of view, it 
is clear that Q1a should be rather short, i.e. $\lambda$ should be rather small. This, however, limits the aperture gain achievable in Q1b due to $r_{\mathrm{g}}$ [see Eq. (33)]. Thus we can expect the optimum split position to be rather in the center of Q1 ( $\lambda$ around 1) than towards the ends (i.e. large or small $\lambda$ ). With this in mind, a good strategy for the optimization of the peak dose is to choose a set of values for $\lambda$ around 1 and optimize $r_{\mathrm{g}}$.

\section{Simulation and results}

To explore the effect of splitting Q1, the FCC-hh interaction region lattice with $L^{*}=36 \mathrm{~m}$ was used. For the first simulations of the radiation load, $\lambda$ was set to 1 . In order to get a realistic design, a gap of $0.64 \mathrm{~m}$ between Q1a and Q1b was introduced. To compensate the slight change in focusing, the triplet was rematched. As a result, the relative change of $k$ of Q1 was less than $10^{-4}$, while the strengths of Q2a/b and Q3 stayed constant. Thus, the radiation optimization has a negligible impact on the beam optics, the minimum beam stay clear was unchanged as it was intended by the parametrization.

Figure 17 shows the peak doses obtained with FLUKA for the lattice with split Q1 for the cases $r_{\mathrm{g}}=1.1$ (V1) and $r_{\mathrm{g}}=1.2$ (V2). Moreover, the unsplit case with a coil aperture of $100 \mathrm{~mm}$ is shown, considering two shielding thickness options: 15 and $20 \mathrm{~mm}$. There is a good agreement of the resulting in Q1a and Q1b with the qualitative predictions in Fig. 16. The dose in Q1a did not increase much, because the shielding in this region actually increased as discussed earlier. For V2, the maximum peak doses in Q1a and Q1b are the same, thus the optimum was found. The optimization in $r_{\mathrm{g}}$ only took two iterations. As the maximum peak dose is now at the beginning of Q2a and the end of Q3, further optimization in $\lambda$ was omitted. The maximum peak dose in Q1 was decreased from about 27 MGy (unsplit, $20 \mathrm{~mm}$ shielding) to about $18 \mathrm{MGy}$, which is a reduction of $\approx 33 \%$. This was achieved with a

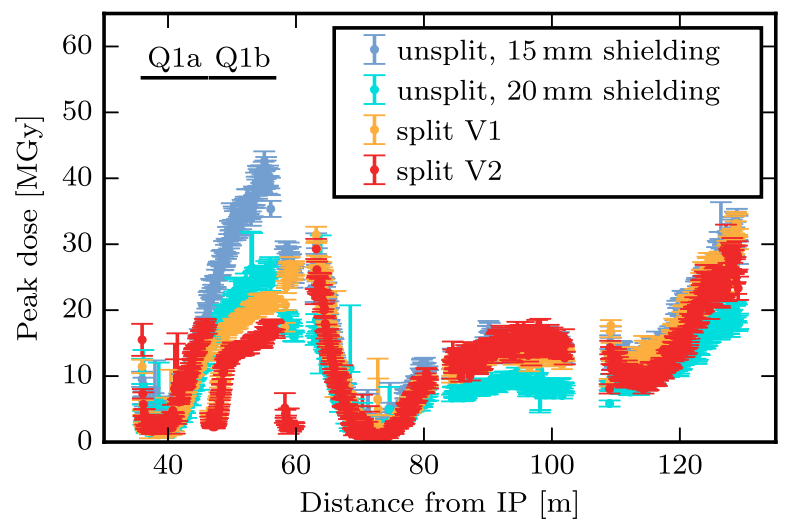

FIG. 17. Peak doses of the triplet with unsplit and split Q1. For an optimized Q1 split (V2), the maximum peak dose is reduced by about $33 \%$ with respect to the unsplit $20 \mathrm{~mm}$ shielding case. shielding thickness of $21 \mathrm{~mm} / 24 \mathrm{~mm}$ and a coil aperture of $92 \mathrm{~mm} / 110 \mathrm{~mm}$ in Q1a and Q1b, respectively. In the rest of the triplet the assumed shielding thickness is $15 \mathrm{~mm}$ and the coil apertures are $100 \mathrm{~mm}$ for $\mathrm{V} 1$ and $115 \mathrm{~mm}$ for V2. Assuming an acceptable dose of $30 \mathrm{MGy}$, the integrated luminosity that Q1 could survive increased to $5000 \mathrm{fb}^{-1}$. This corresponds to the minimum goal for a five-year operation cycle at ultimate parameters [3] allowing to run the full period without replacing Q1.

In order to take full advantage of the dose reduction in Q1, the radiation load in the rest of the triplet needs to be decreased to similar levels. Optimized running scenarios with alternating crossing planes that distribute the radiation azimuthally have shown to be able to reduce the peak doses in the rest of the triplet to about $30 \mathrm{MGy}$ per $4500 \mathrm{fb}^{-1}$ in the $L^{*}=36 \mathrm{~m}$ lattice [30], coming close to the targeted values. In the presence of a horizontal spectrometer, as is the case in the $L^{*}=45 \mathrm{~m}$ lattice, the effectiveness of the crossing angle variation is limited [30]. Instead, alternating the spectrometer polarity is expected to have similar effects.

As mentioned before, surviving $5000 \mathrm{fb}^{-1}$ can only be considered the minimum goal, the number of survivable high luminosity runs will potentially determine the long term performance of FCC-hh. The lattice should be further improved to push towards higher luminosities combined with high survivability.

\section{FIRST DYNAMIC APERTURE STUDIES}

The design strategy deduced in Sec. III D demands to increase the triplet length until limited by chromaticity or dynamic aperture (DA). For this purpose, first exploratory dynamic aperture studies were conducted using SIXTRACK [32]. A first tracking without any magnet errors gave no DA limitation within the probed interval ( $30 \sigma$ to $85 \sigma)$. The DA for collision optics is mainly limited by field errors in the final focus triplet and the separation dipoles, due to the large $\beta$ functions and the orbit offset due to the crossing angle $[33,34]$. In this early study, only the triplet errors are considered. As a first guess for the triplet errors, the error tables and error definitions of the HL-LHC triplet, scaled with the aperture, were used [5]. Dipolar and quadrupolar errors were not considered yet as they are expected to have a minor impact on the DA after correction. The tracking

TABLE V. Parameters of the tracking studies.

\begin{tabular}{lc}
\hline \hline Turns & $10^{5}$ \\
Number of seeds & 60 \\
Normalized emittance & $2.2 \mu \mathrm{m}$ \\
Energy of reference particle $E_{0}$ & $50 \mathrm{TeV}$ \\
Chromaticity $Q^{\prime}$ & 2 \\
Relative momentum spread $d p / p$ & 0.00027 \\
Step size & $2 \sigma$ \\
Particle pairs per step & 30 \\
Angles in x-y plane & 5 \\
\hline \hline
\end{tabular}




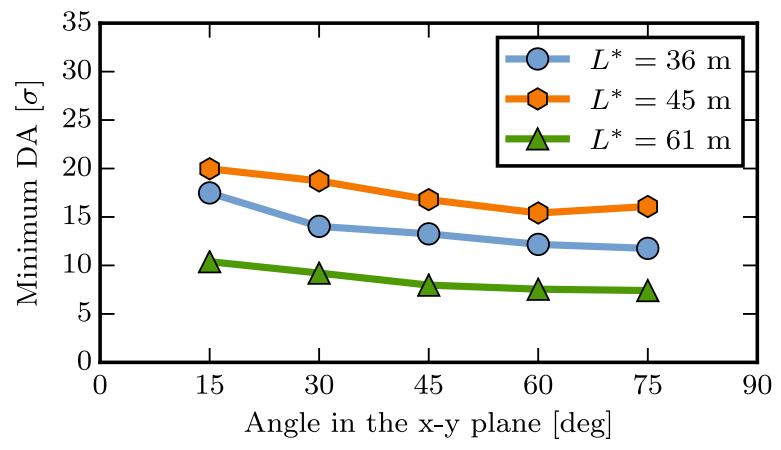

FIG. 18. Minimum dynamic apertures for the IR lattices with $L^{*}=36 \mathrm{~m}, 45 \mathrm{~m}$, and $61 \mathrm{~m}$ with $\beta^{*}=0.3 \mathrm{~m}$. The crossing angles were turned off.

parameters were adopted from first DA studies with main dipole field errors [35] and are listed in Table V.

For the first tracking studies, the crossing angles were turned off, the closed orbit was going through the center of all quadrupoles. The resulting dynamic apertures are plotted in Fig. 18. The $L^{*}=45 \mathrm{~m}$ lattice shows the largest DA around $20 \sigma$. This is likely due to the fact that the field errors scale with the aperture [36]. The fraction of the coil aperture occupied by the beam is the smallest in the $L^{*}=$ $45 \mathrm{~m}$ lattice (the beam stay clear for $\beta^{*}=0.3 \mathrm{~m}$ is $49 \sigma$ without crossing angles, compared to 24 to $26 \sigma$ in the other lattices). Consequently, particles experience the smallest field errors at similar normalized amplitudes. Thus, at constant $\beta^{*}$, the larger aperture due to the longer triplet not only allows for more shielding, but also has a positive impact on the DA. The other two lattices are more comparable in terms of occupied aperture and the $L^{*}=$ $36 \mathrm{~m}$ lattice has a significantly higher DA than the $L^{*}=$ $61 \mathrm{~m}$ lattice. This shows that the $L^{*}$ is a very important parameter for the dynamic aperture and should be chosen as small as possible, affirming the $\beta^{*}$ minimization strategy devised in Sec. III D.

We can expect the crossing angles to worsen the picture as the orbit excursion will increase the beam's sensitivity to errors. Indeed, the DA dropped to $0 \sigma$ when the crossing angles were turned on. The vanishing dynamic aperture indicates that the beam stability in FCC-hh is a larger challenge than in HL-LHC, where studies with the corrector package turned off resulted in a minimum DA of $5 \sigma$ [37].

As a first step to improve the results, an LHC-like local correction of sextupolar errors $\left(a_{3} / b_{3}\right)[33,38,39]$ and a local coupling correction to compensate feed-down effects from field errors were implemented. Figure 19 shows the resulting dynamic aperture. It increased to a minimum of $4 \sigma$ at an angle of $75^{\circ}$. Consequently, the next step for improvement is the correction of higher order multipoles. This was done for octupolar errors $\left(\mathrm{a}_{4} / \mathrm{b}_{4}\right)$ and the normal dodecapolar component $\left(b_{6}\right)$ in Fig. 20. The minimum DA increased to about $7 \sigma$. However further developments in the

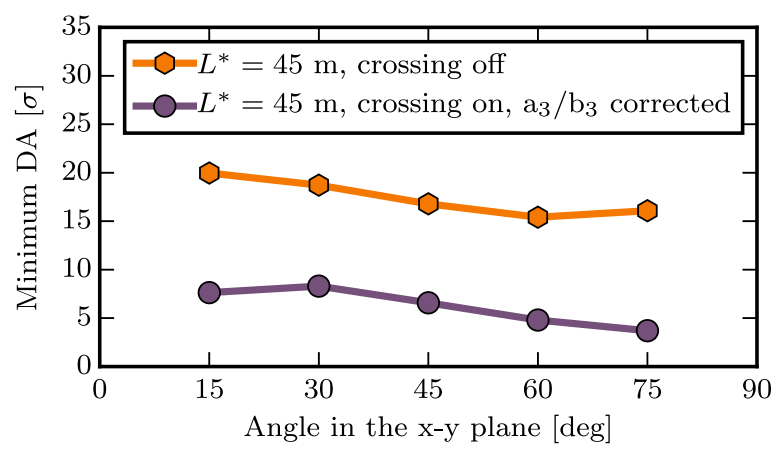

FIG. 19. Minimum dynamic apertures for the $L^{*}=45 \mathrm{~m}$ lattice with $\beta^{*}=0.3 \mathrm{~m}$.

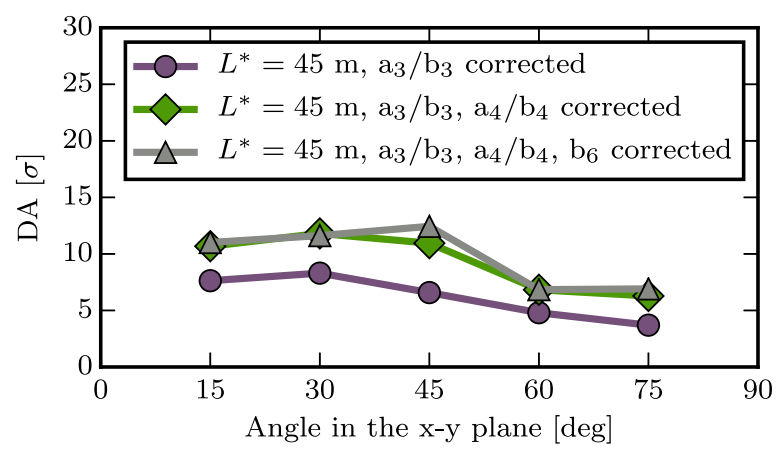

FIG. 20. Minimum dynamic apertures for the $L^{*}=45 \mathrm{~m}$ lattice with $\beta^{*}=0.3 \mathrm{~m}$ for different local multipolar corrections.

correction scheme might be required to improve the DA along the vertical axis.

\section{CONCLUSION AND OUTLOOK}

Starting the design of the FCC-hh interaction region from scaled LHC and HL-LHC lattices, we discovered that the final focus system would be exposed to radiation from collision debris that exceeds the quench limit by 1 order of magnitude and the current lifetime dose limit by 2 orders of magnitude. It is clear that energy deposition is the main driver of the final focus design that needs to combine high luminosity performance with sufficient radiation mitigation. Several lattice options were developed that allowed $\beta^{*}$ values around or below the current ultimate goal of $0.3 \mathrm{~m}$, while leaving space for shielding to protect the magnets. The key figures of the different lattice options are summarized in Table VI. Studies of the minimum $\beta^{*}$ showed a beneficial effect of long triplet magnets while $L^{*}$ only had a minor impact on the limitation of $\beta^{*}$ by the magnet apertures. Still, $L^{*}$ should be kept as small as possible in order to limit chromaticity and the impact of field errors.

A new concept for radiation damage mitigation by splitting the first final focus quadrupole is presented. It is shown to be capable of reducing the peak dose in the most affected area by about $33 \%$. In combination with crossing plane variation, the $L^{*}=36 \mathrm{~m}$ option reaches a sustainable 
TABLE VI. Summary of the presented triplet options. The shielding thickness defines the minimum $\beta^{*}$. The $\beta^{*}$ used for FLUKA studies was set to the ultimate goal where possible and mainly impacts the dose via the crossing angle.

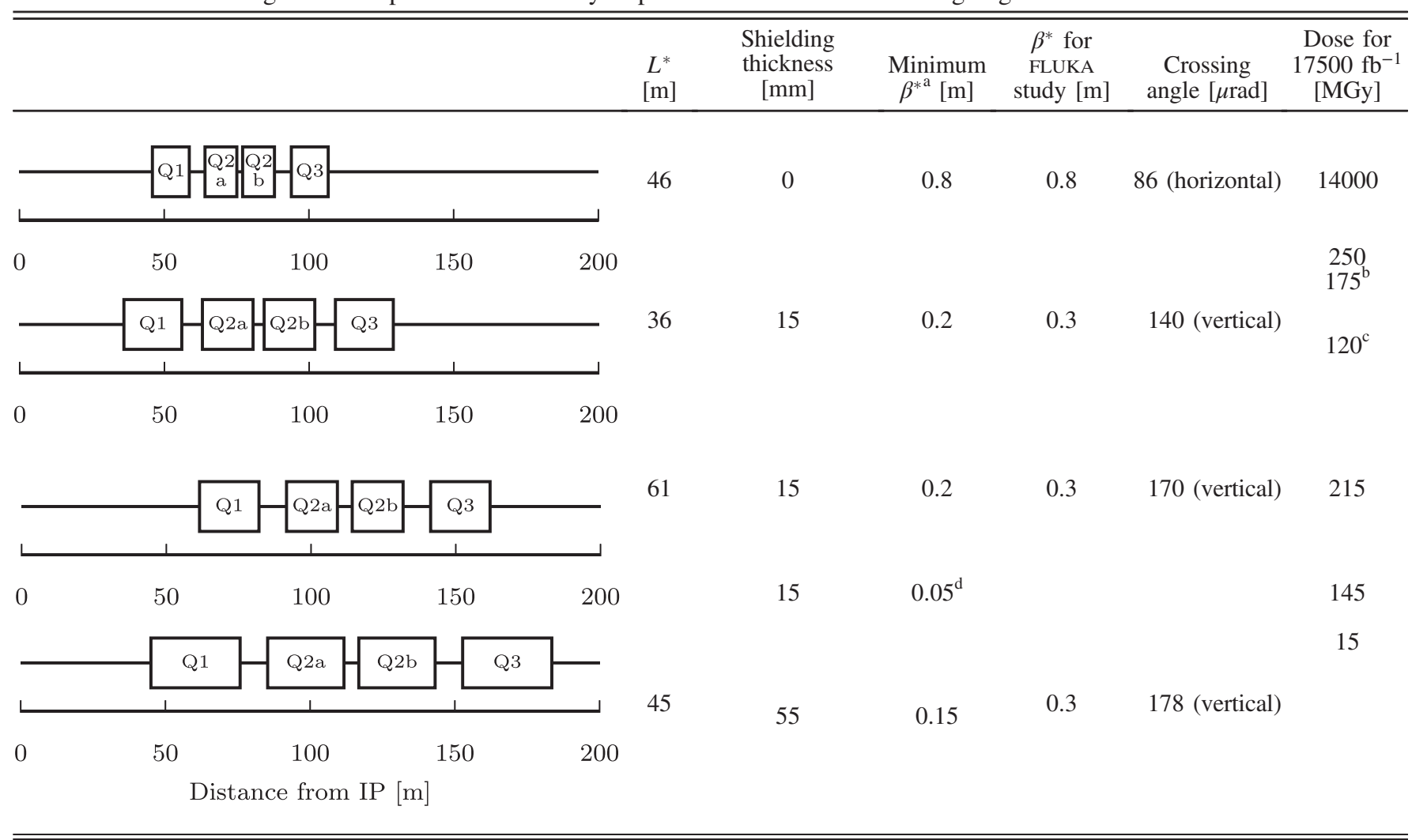

\footnotetext{
${ }^{\mathrm{a}}$ Assuming an ambitious beam stay clear requirement of $12 \sigma$.

${ }^{\mathrm{b}}$ With Q1 split.

${ }^{\mathrm{c}}$ With Q1 split and crossing angle variation.

${ }^{\mathrm{d}}$ Optics solution for arc integration only found for $\beta^{*}=0.1 \mathrm{~m}$ or larger.
}

integrated luminosity in the order of one high luminosity run, i.e. $5000 \mathrm{fb}^{-1}$. Nevertheless, the quadrupoles ideally should sustain the FCC-hh lifetime goal of $17500 \mathrm{fb}^{-1}$.

While the conventional approach with very thick shielding in the $L^{*}=45 \mathrm{~m}$ lattice already meets this goal, it also limits the achievable minimum $\beta^{*}$ to $0.2 \mathrm{~m}$. The more ambitious option using only moderate shielding thickness but featuring a minimum $\beta^{*}$ down to $0.05 \mathrm{~m}$ motivates research on more radiation resistant magnets. With materials that feature lifetime limits around 100 MGy or more, the sustainable integrated luminosity would already be in the order of the FCC-hh lifetime goal of $17500 \mathrm{fb}^{-1}$ for the $L^{*}=45 \mathrm{~m}$ lattice. This also applies to the more compact $L^{*}=36 \mathrm{~m}$ lattice if mitigation measures are applied.

The crossing angles together with magnet imperfections have shown to be the biggest challenge for the dynamic aperture at $\beta^{*}=0.3 \mathrm{~m}$. With a full local correction of sextupolar and octupolar triplet errors, a DA of $7 \sigma$ was found for the $L^{*}=45 \mathrm{~m}$ lattice. This value is rather encouraging for this early design phase. However, a value of about $12 \sigma$ seems feasible with further development of the correction scheme. Furthermore, the vanishing of the dynamic aperture without local correction poses an operational challenge and needs to be understood.

Based on these studies, the $L^{*}=45 \mathrm{~m}$ lattice has become the reference design for the interaction region of FCC-hh. In the next steps, the lattice needs to become more realistic, specifically in the maximum lengths of individual magnets and in the spacing between them.

The impact of alternative detector designs, e.g. with a forward solenoid instead of the spectrometer dipole, has to be studied.

\section{ACKNOWLEDGMENTS}

We would like to thank A. Chancé for collaboration with the lattice integration as well as E. H. Maclean for helping to implement his LHC correction scripts in FCC-hh. We further thank D. Schulte for suggesting the scaling strategy leading to $L^{*}=61 \mathrm{~m}$ and $\mathrm{E}$. Todesco for discussions on magnet related issues. This work is supported by the Wolfgang Gentner Programme of the Federal Ministry of Education and Research, Germany (BMBF). 
[1] ATLAS and CMS Collaborations, Technical Report No. ATLUPGRADE-PUB-2013-014, CERN, Geneva, 2013.

[2] D. Schulte et al., Technical Report No. FCC-ACC-SPC0001, EDMS No. 1342402, CERN, Geneva, 2014.

[3] D. Schulte, Deliverable Report No. EuroCirCol-D1-1, CERN, 2015.

[4] A. Apollonio, LHC Report No. CERN-BULL-2016-28-29, CERN, 2016.

[5] High-Luminosity Large Hadron Collider (HL-LHC). Preliminary Design Report, edited by G. Apollinari, I. Bájar Alonso, O. Brüning, M. Lamont, and L. Rossi, CERN, Geneva, 2015.

[6] G. Aad et al. (ATLAS Collaboration), The ATLAS experiment at the CERN Large Hadron Collider, J. Instrum. 3, S08003 (2008).

[7] LHC Design Report, edited by O. S. Brüning, P. Collier, P. Lebrun, S. Myers, R. Ostojic, J. Poole, and P. Proudlock, CERN, Geneva, 2004.

[8] J.-P. Koutchouk, A. Faus-Golfe, A. Verdier, and S. Weisz, Modular optical design of the LHC experimental insertions, in Proceedings of the 5th European Particle Accelerator Conference (IOP, Bristol, 1996), Vol. 5, p. 911.

[9] R. Tomás, M. Benedikt, A. Bogomyagkov, L. Bottura, F. Cerutti, L. Esposito, A. Ferrari, B. Haerer, B. Holzer, E. Jensen, M. Koratzinos, R. Martin, L. Medina, D. Schulte, E. Todesco, J. Wenninger, S. White, and F. Zimmermann, FCC study: parameters and optics for hadron and lepton colliders, Nucl. Part. Phys. Proc. 273, 149 (2016).

[10] R. Bruce, R. De Maria, S. Fartoukh, M. Giovannozzi, S. Redaelli, R. Tomás, and J. Wenninger, Technical Report No. CERN-ACC-2014-0044, CERN, Geneva, 2014.

[11] M. Fiascaris, R. Bruce, D. Mirarchi, and S. Redaelli, First design of a proton collimation system for $50 \mathrm{TeV}$ FCC-hh, in Proceedings of the 7th International Particle Accelerator Conference (IPAC'16), Busan, Korea, 2016 (JACoW, Geneva, Switzerland, 2016), pp. 2423-2426.

[12] R. de Maria, General method for final focus system design for circular colliders, Phys. Rev. ST Accel. Beams 11, 031001 (2008).

[13] W. Herr and T. Pieloni, Beam-Beam effects, arXiv:1601.05235.

[14] F. Antoniou et al., LHC Luminosity modeling for RUNII, in Proceedings of the 7th International Particle Accelerator Conference (IPAC'16), Busan, Korea, 2016 (JACoW, Geneva, 2016), pp. 1403-1406.

[15] A. Ferrari, P. R. Sala, A. Fassò, and J. Ranft, FLUKA: A Multiparticle Transport Code (program version 2005) (CERN, Geneva, 2005).

[16] G. Battistoni et al., Pluri- and trans-disciplinarity, towards new modeling and numerical simulation paradigms, Joint International Conference on Supercomputing in Nuclear Applications and Monte Carlo 2013, SNA+MC 2013 [Ann. Nucl. Energy 82, 10 (2015)].

[17] V. Boccone, R. Bruce, M. Brugger, M. Calviani, F. Cerutti, L. S. Esposito, A. Ferrari, A. Lechner, A. Mereghetti, E. Nowak, N. V. Shetty, E. Skordis, R. Versaci, and V. Vlachoudis, Beam-machine Interaction at the CERN LHC, Nucl. Data Sheets 120, 215 (2014).

[18] S. Roesler, R. Engel, and J. Ranft, The Monte Carlo event generator DPMJET-III, in Advanced Monte Carlo for radiation physics, particle transport simulation and applications, in Proceedings Conference, MC2000, Lisbon, Portugal, 2000, arXiv:hep-ph/0012252, pp. 1033-1038.

[19] A. Fedynitch and R. Engel, Revision of the high energy hadronic interaction models PHOJET/DPMJET-III, in Proceedings of the 14th International Conference on Nuclear Reaction Mechanisms, Varenna, Italy, 2015 (CERN, Geneva, 2015), pp. 291-300.

[20] D. Tommasini (personal communication).

[21] W. Riegler, FCC-hh detector overview, at FCC Week, 2015.

[22] F. Broggi, Energy deposition in the triplet and TAS issues, in Proceedings of the CARE-HHH-APD Workshop on interaction regions for the LHC upgrade, DAFNE, and SuperB “IR07" (CERN, Geneva, 2008).

[23] C. Hoa, F. Cerutti, and E. Wildner, Technical Report No. LHC-PROJECT-Report-1167; Report No. CERNLHC-PROJECT-Report-1167, CERN, Geneva, 2008.

[24] D. Schoerling, Review of average/peak power limits for high-luminosity IR triplet magnets, at the 1st FCC-hh other magnet design meeting, 2017.

[25] MAD-Methodical accelerator design, http://mad.web .cern.ch/mad/.

[26] L. Bottura and P. Fessia, What could stop us and when, in RLIUP: Review of LHC and injector upgrade plans, 2014 (CERN, Geneva, 2014).

[27] W. Riegler, FCC-hh experiments and detectors overview, at FCC week, 2016.

[28] M. Besana, F. Cerutti, A. Ferrari, W. Riegler, and V. Vlachoudis, Characterization of the radiation field in the FCC-hh detector, in Proceedings of the 7th International Particle Accelerator Conference (IPAC'16), Busan, Korea, 2016 (Ref. [11]), pp. 1414-1417.

[29] X. Buffat, Beam parameters evolution and luminosity performance, at FCC week, 2016.

[30] M. Besana, F. Cerutti, S. Fartoukh, R. Martin, and R. Tomás, Assessment and mitigation of the proton-proton collision debris impact on the FCC triplet, in Proceedings of the 7th International Particle Accelerator Conference (IPAC'16), Busan, Korea, 2016 (JACoW, Geneva, 2016), pp. 1410-1413.

[31] R. Martin, M. Besana, F. Cerutti, and R. Tomás, Radiation load optimization in the final focus system of FCC-hh, in Proceedings of the 7th International Particle Accelerator Conference (IPAC'16), Busan, Korea, 2016 (Ref. [11]), pp. 1462-1465.

[32] SixTRACK -6D tracking code, http://sixtrack.web.cern.ch/ SixTrack/.

[33] E. H. Maclean, R. Tomás, M. Giovannozzi, and T. H. B. Persson, First measurement and correction of nonlinear errors in the experimental insertions of the CERN Large Hadron Collider, Phys. Rev. ST Accel. Beams 18, 121002 (2015).

[34] O.S. Brüning and S.D. Fartoukh, Technical Report No. LHC-Project-Report-501; Report No. CERN-LHCProject-Report-501, CERN, Geneva, 2001.

[35] B. Dalena et al., First evaluation of dynamic aperture at injection for FCC-hh, in Proceedings of the 7th International Particle Accelerator Conference (IPAC'16), Busan, Korea, 2016 (Ref. [11]), pp. 1466-1469. 
[36] E. Todesco, B. Bellesia, J.-P. Koutchouk, and C. Santoni, Technical Report No. CERN-LHC-PROJECT-Report1061, CERN, 2007.

[37] M. Giovannozzi, R. D. Maria, and S. Fartoukh, Specification of a system of correctors for the triplets and separation dipoles of the LHC upgrade, in Proceedings of the 4th International Particle Accelerator Conference (IPAC'13), Shanghai, China, 2013 (JACoW, Geneva, Switzerland, 2013), pp. 2612-2614.
[38] O. S. Brüning, S. D. Fartoukh, M. Giovannozzi, and T. Risselada, Technical Report No. LHC-Project-Note-349, CERN, Geneva, 2004.

[39] R. Tomás, M. Giovannozzi, and R. de Maria, Nonlinear correction schemes for the phase 1 LHC insertion region upgrade and dynamic aperture studies, Phys. Rev. ST Accel. Beams 12, 011002 (2009). 